\title{
Universal quantum gates, artificial neurons, and pattern recognition simulated by $L C$ resonators
}

\author{
Motohiko Ezawa (ㄷ) \\ Department of Applied Physics, University of Tokyo, Hongo 7-3-1, 113-8656, Japan
}

(Received 10 December 2020; revised 3 March 2021; accepted 22 March 2021; published 16 April 2021)

\begin{abstract}
We propose to simulate quantum gates by $L C$ resonators, where the amplitude and the phase of the voltage describe the quantum state. By controlling the capacitance or inductance of resonators, it is possible to control the phase of the voltage arbitrarily. A set of resonators acts as the phase-shift, the Hadamard, and the CNOT gates. They constitute a set of universal quantum gates. We also discuss an application to an artificial neuron. As an example, we study pattern recognition of numbers and alphabets by evaluating the similarity between an input and the reference pattern. We also study colored pattern recognition by using a complex neural network.
\end{abstract}

DOI: 10.1103/PhysRevResearch.3.023051

\section{INTRODUCTION}

Quantum computation is one of the most exciting fields of current physics [1-3]. It is realized in various systems including superconductors [4], photonic systems [5], quantum dots [6], trapped ions [7], and nuclear magnetic resonance [8,9]. For universal quantum computation, it is well known that only three quantum gates are enough, which are the phase-shift, the Hadamard, and the CNOT gates [10-12].

There are also some attempts to simulate various quantum gates by electric circuits [13-15], motivated by recent development of topological physics with the use of electric circuits [16-29]. Among them, a network of telegrapher lines is capable to simulate the universal quantum gates $[13,14]$, because the Kirchhoff law may be rewritten in the form of the Schrödinger equation [30]. This formulation requires long wires for a long quantum algorithm, where quantum states evolve spatially from the left wires to the right wires.

Quantum computation may be indispensable to quantum machine learning, which is an emerging field of contemporary physics [31-42]. Neural networks are often used in machine learning, where artificial neurons are basic elements [43,44]. An artificial neuron has an internal degree of freedom called the weight. The output is determined from the input data by taking the inner product between the input data and the weight, and by applying an activation function to it. The inner product of two objects measures the similarity between them. For instance, using a series of numbers representing a reference pattern as the weight, we may analyze the similarity between an input pattern and the reference pattern as an output. Artificial neuron is simulated by quantum computer [45-51]. Taking the inner product is the heaviest process, which will be executable by a quantum computer [48,52].

In this paper, we propose to simulate a quantum computer by a set of $L C$ resonators, where a set of voltage and current

Published by the American Physical Society under the terms of the Creative Commons Attribution 4.0 International license. Further distribution of this work must maintain attribution to the author(s) and the published article's title, journal citation, and DOI. represents a wave function. First, we construct a phase-shift gate with an arbitrary phase by tuning the capacitance of an $L C$ resonator. Next, we construct the Hadamard gate by tuning the inductance of an inductor bridging two $L C$ resonators. We also construct the CNOT and the controlled phase-shift gate by using a voltage-controlled inductor or capacitor.

We then discuss applications to artificial neuron and pattern recognition. The calculation of the inner product may be executed by the operation of $L C$ resonators for arbitrary inputs and weights. We elucidate the difference between the standard quantum-circuit implementation and the present electric-circuit implementation of the inner product. As a merit of the present scheme, it may directly deal with complex numbers by using the amplitude and the phase delay of the voltage. Furthermore, although it is not scalable, it would be easier to make a hybrid of small-size quantum computer and classical computer in the present approach since both of them use electric circuits.

This paper is composed as follows. In Sec. II, we start with a discussion how to store the information of $N$ qubits $|j\rangle\rangle \equiv$ $\left|n_{1} n_{2} \cdots n_{N}\right\rangle$ in a set of $L C$ resonators, where $n_{i}=0,1$. Then, we propose to construct various quantum gates including a set of universal quantum gates by $L C$ resonators.

In Sec. III, we apply our formalism to study artificial neurons, where we express various data with the aid of so-called real equally weighted (REW) states [53-55]. They are superposition states of $N$-qubits with coefficients $\alpha_{j}= \pm 1 / \sqrt{2^{N}}$. In Sec. IV, we discuss pattern recognition by calculating the inner product of an input data and the reference data. We present explicit examples of number recognition and alphabet recognition.

In Sec. $\mathrm{V}$, we generalize REW states to include complex coefficients $\alpha_{j}=e^{i \theta_{j}} / \sqrt{2^{N}}$. We call them complex equally weighted (CEW) states. Then, we introduce complex-artificial neurons to deal with the inner product of CEW states. In Sec. VI, we propose to represent a colored pattern by a CEW state, where colored pattern recognition is done by evaluating the inner product of two CEW states representing the reference and an input pattern.

In Sec.VII, we present an electric-circuit implementation of quantum gates for calculation of an inner product starting 
(a) Two Qubits

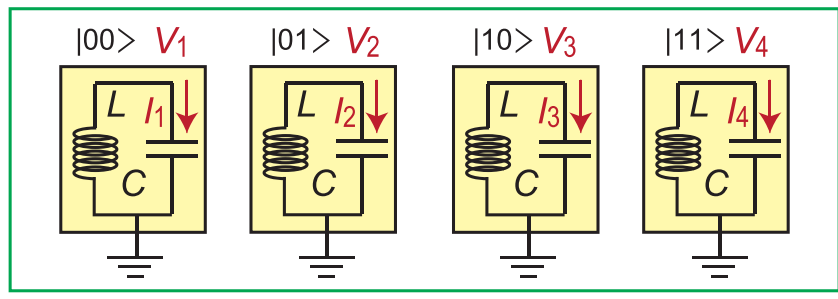

(b) Phase-shift gate

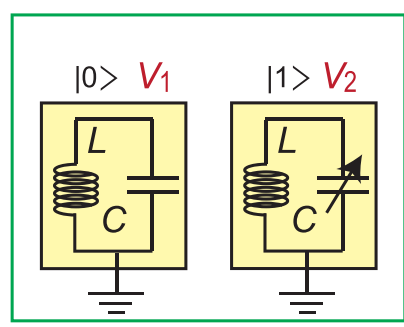

(c) Mixing gate

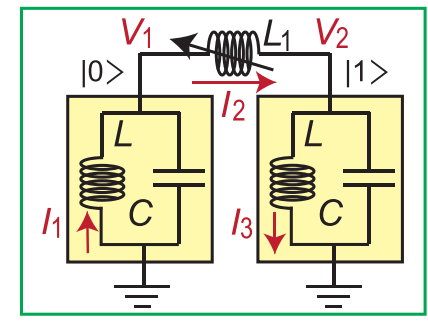

(d) CNOT gate

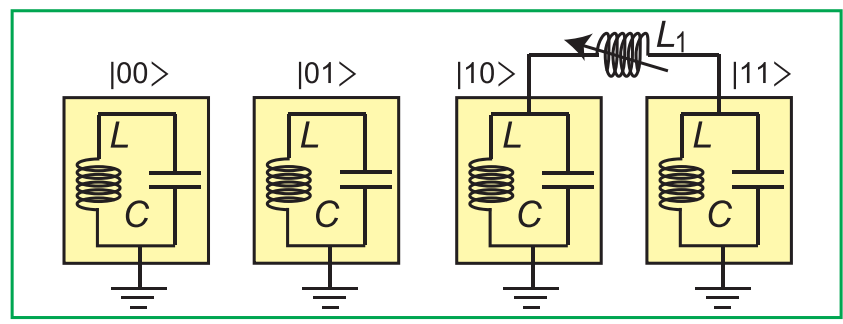

(e) Controlled phase-shift gate

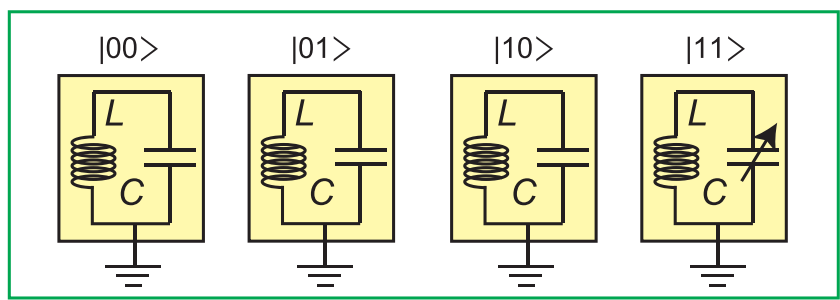

FIG. 1. (a) Two qubits made of four $L C$ resonators. (b) Phaseshift gate consisting a pair of $L C$ resonators. The capacitance of the state $|1\rangle$ is controlled. (c) Mixing gate. The inductance of the inductor $L_{1}$ bridging two resonators is controlled. The Hadamard gate is constructed by a combination of the mixing gate and the phase-shift gate. (d) CNOT gate. We bridge the resonators representing $|10\rangle$ and $|11\rangle$ by the inductor $L_{1}$ (e) Controlled phase-shift gate. We control the capacitance of the resonator representing $|11\rangle$.

from the initial $N$-qubit state $|00 \cdots 0\rangle$. In Sec.VIII, we explore REW states from a viewpoint of graph and hypergraph states. We also introduce weighted graph and hypergraph states to represent CEW states. In Sec. IX, we discuss merits and demerits of the present approach.

\section{LC RESONATORS, QUBITS, AND GATES}

We use a set of $2^{N}$ identical $L C$ resonators to simulate $N$-qubit quantum computation. An instance of $N=2$ is illustrated in Fig. 1(a). The voltage of the $j$ th $L C$ resonator is expressed as

$$
V_{j}(t)=V_{j}^{0} \cos \left(\omega_{0} t+\theta_{j}\right)
$$

where $\omega_{0}=1 / \sqrt{L C}$ is the resonant frequency, $V_{j}^{0}$ is the absolute value of the voltage, and $\theta_{j}$ is the phase shift.

A qubit state is defined by a superposition of the two states $|0\rangle$ and $|1\rangle$ as $|\psi\rangle=\alpha_{0}|0\rangle+\alpha_{1}|1\rangle$. Similarly, an $N$-qubit state is defined by a superposition of the $2^{N}$ states as

$$
|\psi\rangle=\sum_{n_{j}=0,1} \alpha_{n_{1} n_{2} \cdots n_{N}}\left|n_{1} n_{2} \cdots n_{N}\right\rangle
$$

which is expressed equivalently as

$$
\left.|\psi\rangle=\sum_{j=0}^{2^{N}-1} \alpha_{j}|j\rangle\right\rangle,
$$

where $j$ is the decimal number corresponding to the binary number $\left(n_{1} n_{2} \cdots n_{N}\right)$ such as $\left.\left.|0\rangle\right\rangle=|0 \cdots 00\rangle,|1\rangle\right\rangle=$ $\left.|0 \cdots 01\rangle, \cdots,\left|2^{N}-1\right\rangle\right\rangle=|11 \cdots 1\rangle$.

It is a key observation [15] that we may set

$$
\alpha_{j}=V_{j}^{0} e^{i \theta_{j}} / \sqrt{\sum_{j}\left(V_{j}^{0}\right)^{2}}
$$

in the $L C$-resonator realization of quantum computation. Thus we store the information of $N$ qubits in a set of $L C$ resonators.

Here we propose to carry out a gate process by controlling externally the value $C$ of a capacitance as in Fig. 1(b) or the value $L_{1}$ of an inductor bridging two $L C$ resonators as in Fig. 1(c). For each gate process, the initial and the final systems are the same set of $2^{N}$ identical $L C$ resonators with the same energy, although the coefficient $\alpha_{j}$ may be modified for some $j$. A gate process is required to be adiabatic.

The gate $U$ is represented by a $2^{N} \times 2^{N}$ matrix $U_{j k}$ such that

$$
\left.U|j\rangle\rangle=\sum_{k=0}^{2^{N}-1} U_{j k}|k\rangle\right\rangle .
$$

By this operation, the initial state $\psi^{\text {ini }}$ is brought to the final state $\psi^{\text {fin }}=U \psi^{\text {ini }}$, where $\left.\psi^{\text {ini }}=\sum_{j=0}^{2^{N}-1} \alpha_{j}^{\text {ini }}|j\rangle\right\rangle$ and $\psi^{\text {fin }}=$ $\left.\sum_{k=0}^{2^{N}-1} \alpha_{k}^{\text {fin }}|k\rangle\right\rangle$. It follows that

$$
\alpha_{k}^{\mathrm{fin}}=\sum_{j=0}^{2^{N}-1} \alpha_{j}^{\mathrm{ini}} U_{j k}=\sum_{j=0}^{2^{N}-1} U_{k j} \alpha_{j}^{\mathrm{ini}},
$$

since $U$ is a symmetric matrix in universal quantum computation.

Kirchhoff law and Schrödinger equation. The Kirchhoff law for a set of independent $L C$ resonators may be rewritten in the form of the Schrödinger equation [13,30],

$$
i \frac{d}{d t} \psi_{j}=H \psi_{j}
$$

where $H(t)=-\omega_{0} \sigma_{y}$ is the Hamiltonian, and

$$
\psi_{j}=\left(\mathcal{I}_{j}, \mathcal{V}_{j}\right)^{t}=\left(\sqrt{L / C} I_{j}, V_{j}\right)^{t}
$$

is the wave function associated with the $j$ th $L C$ resonator with $I_{j}$ and $V_{j}$ being the current and voltage as indicated in Fig. 1(a) in an instance of two qubits. 
Energy conservation and probability conservation. The total energy of the system is given by $U_{\mathrm{T}}=U_{\mathrm{E}}+U_{\mathrm{M}}$ with

$$
U_{\mathrm{E}}=\frac{C}{2} \sum_{j} V_{j}^{2}, \quad U_{\mathrm{M}}=\frac{L}{2} \sum_{j} I_{j}^{2},
$$

where $U_{\mathrm{E}}$ and $U_{\mathrm{M}}$ are the electrostatic energy and the magnetic energy, respectively.

On the other hand, by using Eq. (8), the probability of the wave function is rewritten as

$$
\sum_{j}\left|\psi_{j}\right|^{2}=\sum_{j} \mathcal{I}_{j}^{2}+\mathcal{V}_{j}^{2}=\sum_{j} \frac{L}{C} I_{j}^{2}+V_{j}^{2}=\frac{2}{C} U_{\mathrm{T}} .
$$

Hence, the conservation of the probability of the wave function is assured by the conservation of the total energy [14]. As we have stated, we arrange a gate process so that the total energy is the same before and after the gate process. It corresponds to the conservation of the probability for qubits $\sum_{j}\left|\alpha_{j}\right|^{2}=1$.

Phase-shift gate. The phase-shift gate is defined by the matrix

$$
U_{\phi}=\left(\begin{array}{cc}
1 & 0 \\
0 & e^{i \phi}
\end{array}\right)
$$

which acts on the one-qubit state $(|0\rangle,|1\rangle)^{t}$. Namely, the action is

$$
U_{\phi}|0\rangle=|0\rangle, \quad U_{\phi}|1\rangle=e^{i \phi}|1\rangle .
$$

To generate the phase shift $\phi$ in the wave function, it is enough to control only the capacitance $C$ in the $L C$ resonator externally during the gating process as shown in Fig. 1(b). We control the capacitance as $C(t)=C_{0}+C_{1}(t)$, where

$$
C_{1}(t)=\frac{C_{1}^{0}}{2}\left(\tanh \frac{t-t_{1}}{T}-\tanh \frac{t-t_{2}}{T}\right),
$$

with four parameters $C_{1}^{0}, t_{1}, t_{2}$ and $T$, as shown in Figs. 2(a1), 2(b1), and 2(c1).

It is possible to determine analytically how the phase shift $\phi$ depends on these parameters by calculating the Berry phase. Since the voltage evolution $V(t)$ is written as a Schrödinger equation, we may use an adiabatic approximation. The snap shot wave function at time $t=\tau$ is given by $\psi(\tau)=\sqrt{2 L / C} I_{0} \bar{\psi}(\tau)$, where $\bar{\psi}(\tau)$ is the normalized wave function

$$
\bar{\psi}(\tau)=\frac{1}{\sqrt{2}} \exp \left(i \omega_{\tau} \tau\right)\left(\begin{array}{c}
1 \\
-i
\end{array}\right),
$$

with $\omega_{\tau}$ the snapshot frequency

$$
\omega_{\tau}=1 / \sqrt{L C(\tau)}
$$

The Berry phase is calculated as

$$
\begin{aligned}
\gamma & =i \int_{0}^{t}\left\langle\bar{\psi}(\tau)\left|\partial_{\tau}\right| \bar{\psi}(\tau)\right\rangle d \tau \\
& =\int_{0}^{t} \frac{1}{2 \sqrt{L} C(\tau)^{3 / 2}}\left(2 C(\tau)-\tau \frac{d C(\tau)}{d \tau}\right) d \tau \\
& =\int_{0}^{t}\left[\omega(\tau)-\frac{\tau}{\sqrt{L} C(\tau)^{3 / 2}} \frac{d C(\tau)}{d \tau}\right] d \tau .
\end{aligned}
$$
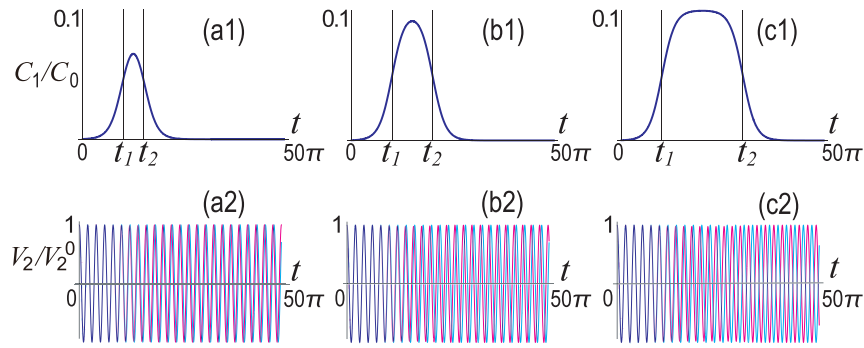

(a3)
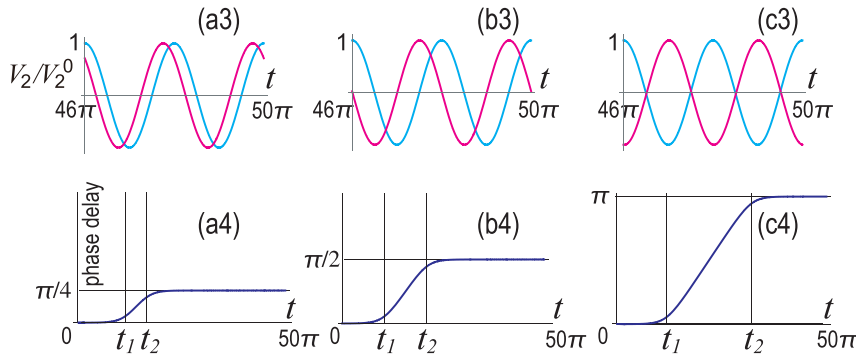

FIG. 2. Phase-shift gate [Fig. 1(b)] for (a) $\phi=\pi / 4$, (b) $\phi=\pi / 2$ and (c) $\phi=\pi$. The phase delay is controlled by a time duration of the capacitance perturbation $C_{1}(t)$. (1) Time evolution of the perturbed capacitance. (2) Time evolution of the voltage $V_{2}$. The voltage $V_{2}$ with (without) the perturbation is represented by a magenta (cyan) curve. (3) Voltage $V_{2}$ in the final states. (4) Phase delay as a function of time $t$. The horizontal axis is time $t$. Time span is $0<t<50 \pi$ for (1), (2), and (4). It is $46 \pi<t<50 \pi$ for (*3), representing the final state. We set $C_{1}^{0} / C_{0}=0.1$ and $T=10 \omega_{0}$ for $0<\omega_{0} t<50 \pi$. We set $\omega_{0} t_{1}=10 \pi$ for all phase-shift gate. We also set $\omega_{0} t_{2}=15 \pi$ for the $\pi / 4$ phase-shift gate, $\omega_{0} t_{2}=20 \pi$ for the $\pi / 2$ phase-shift gate and $\omega_{0} t_{2}=30 \pi$ for the $\pi$ phase-shift (i.e., Pauli Z) gate,

When the perturbation $C_{1}(t)$ is small enough with respect to $C_{0}$, it is calculated as

$$
\gamma \simeq i \omega_{0} t-\phi
$$

up to the first order of $C_{1}(t)$, where $\phi$ is the phase shift given by

$$
\begin{aligned}
\phi= & \omega_{0} \int_{0}^{t} \frac{C_{1}(\tau)}{2 C} d \tau \\
=\omega_{0} & \frac{C_{1}^{0}}{2 C} T\left(\ln \cosh \frac{t-t_{1}}{T}-\ln \cosh \frac{t_{1}}{T}\right. \\
& \left.\quad-\ln \cosh \frac{t-t_{2}}{T}+\ln \cosh \frac{t_{2}}{T}\right) .
\end{aligned}
$$

It is approximated as

$$
\phi=\frac{C_{1}^{0} \omega_{0}}{2 C}\left(t_{2}-t_{1}\right),
$$

provided $T \ll t_{1}<t_{2} \ll t$. Hence, we can tune the phase shift $\phi$ arbitrary by controlling the magnitude of $\left(t_{2}-t_{1}\right) C_{1}^{0}$.

We next solve numerically the differential equation (7) to study the time evolution of the voltage $V(t)$, and confirm the phase-shift formula (19). When we fix $C_{1}^{0}=0.1 C_{0}$ and $\omega_{0} t_{1}=$ $10 \pi$, we have

$$
\phi=\frac{1}{20}\left(\omega_{0} t_{2}-10 \pi\right) .
$$

We present numerical results of the time evolution $V(t)$ by choosing $\omega_{0} t_{2}=15 \pi, 20 \pi$, and $30 \pi$ in Figs. 2(a2), 2(b2), and 
2(c2). See Figs. 2(a3), 2(b3), and 2(c3) for $V(t)$ for $t \gg t_{2}$, representing the final state. The phase shift is found to occur due to the perturbation $C_{1}(t)$. The phase shift $\phi(t)$ during a gating process is shown in Figs. 2(a4), 2(b4), and 2(c4). After the gating process, the resonance frequency returns to $\omega_{0}$ but the phase $\phi$ becomes different from the initial value. It reads $\phi=\pi / 4, \pi / 2$ and $\pi$ as in Figs. 2(a4), 2(b4), and 2(c4). These numerical results confirm the analytical formula (19).

Hadamard gate. The Hadamard gate is defined by the matrix

$$
U_{\mathrm{H}} \equiv \frac{1}{\sqrt{2}}\left(\begin{array}{cc}
1 & 1 \\
1 & -1
\end{array}\right),
$$

which acts on the one-qubit state $(|0\rangle,|1\rangle)^{t}$. It is known to be given by $[13,14]$

$$
U_{\mathrm{H}}=e^{-i \pi / 4} U_{\pi / 2} U_{\text {mix }} U_{\pi / 2},
$$

where $U_{\pi / 2}$ is the $\pi / 2$ phase-shift gate, while $U_{\text {mix }}$ is the mixing gate defined by

$$
U_{\operatorname{mix}}=\frac{1}{\sqrt{2}}\left(\begin{array}{cc}
e^{i \pi / 4} & e^{-i \pi / 4} \\
e^{-i \pi / 4} & e^{i \pi / 4}
\end{array}\right) .
$$

We construct the mixing gate (23) in what follows.

We set a pair of $L C$ resonators bridged by an inductor $L_{1}$ as shown in Fig. 1(c), where the inductance $L_{1}$ is controlled externally. The Kirchhoff law reads

$$
\frac{d}{d t}\left(\begin{array}{l}
I_{1} \\
I_{2} \\
I_{3} \\
V_{1} \\
V_{2}
\end{array}\right)=\left(\begin{array}{ccccc}
0 & 0 & 0 & \frac{1}{L} & 0 \\
0 & 0 & 0 & -\frac{1}{L_{1}} & \frac{1}{L_{1}} \\
0 & 0 & 0 & 0 & -\frac{1}{L} \\
-\frac{1}{C} & \frac{1}{C} & 0 & 0 & 0 \\
0 & -\frac{1}{C} & \frac{1}{C} & 0 & 0
\end{array}\right)\left(\begin{array}{l}
I_{1} \\
I_{2} \\
I_{3} \\
V_{1} \\
V_{2}
\end{array}\right),
$$

where $I_{1}, I_{2}, I_{3}, V_{1}$, and $V_{2}$ are defined in Fig. 1(c). It is rewritten in the form of the Schrödinger equation as in Eq. (7) with the Hamiltonian

$$
H=\frac{1}{\sqrt{L C}}\left(\begin{array}{ccccc}
0 & 0 & 0 & i & 0 \\
0 & 0 & 0 & -i L / L_{1}(t) & i L / L_{1}(t) \\
0 & 0 & 0 & 0 & -i \\
-i & i & 0 & 0 & 0 \\
0 & -i & i & 0 & 0
\end{array}\right),
$$

and the wave function

$$
\left(\mathcal{I}_{1}, \mathcal{I}_{2}, \mathcal{I}_{3}, \mathcal{V}_{1}, \mathcal{V}_{2}\right)=\left(\sqrt{\frac{L}{C}} I_{1}, \sqrt{\frac{L}{C}} I_{2}, \sqrt{\frac{L}{C}} I_{3}, V_{1}, V_{2}\right) .
$$

By making a snapshot approximation, the eigenvalues are given by

$$
E=0, \pm \omega_{0}, \pm \ell(t) \omega_{0},
$$

with

$$
\ell(t)=\sqrt{1+2 L / L_{1}(t)}
$$

at each $t$.

We consider a process where the inductor $L_{1}$ is bridged to the $L C$ resonators during a time interval $t_{1}<t<t_{2}$ but not for $t<t_{1}$ and $t>t_{2}$ in Fig. 1(c). For example, we take

$$
\frac{1}{L_{1}(t)}=\frac{1}{2 L_{1}}\left(\tanh \frac{t-t_{1}}{T}-\tanh \frac{t-t_{2}}{T}\right),
$$

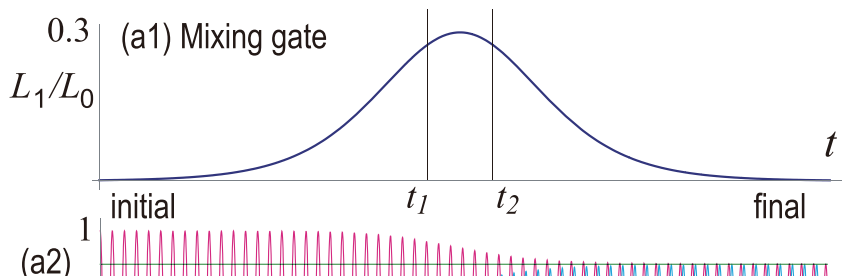

(a2)

$V / V^{0}$

$V / V^{0}$
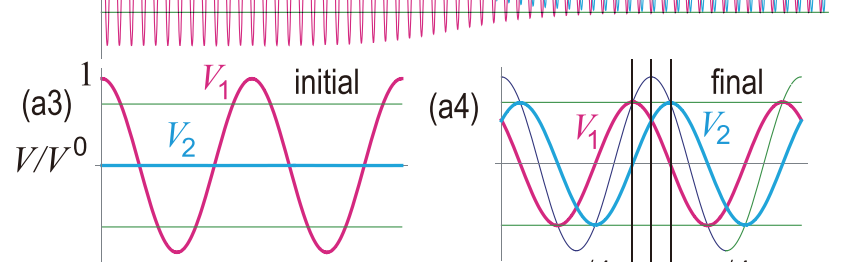

(a4)
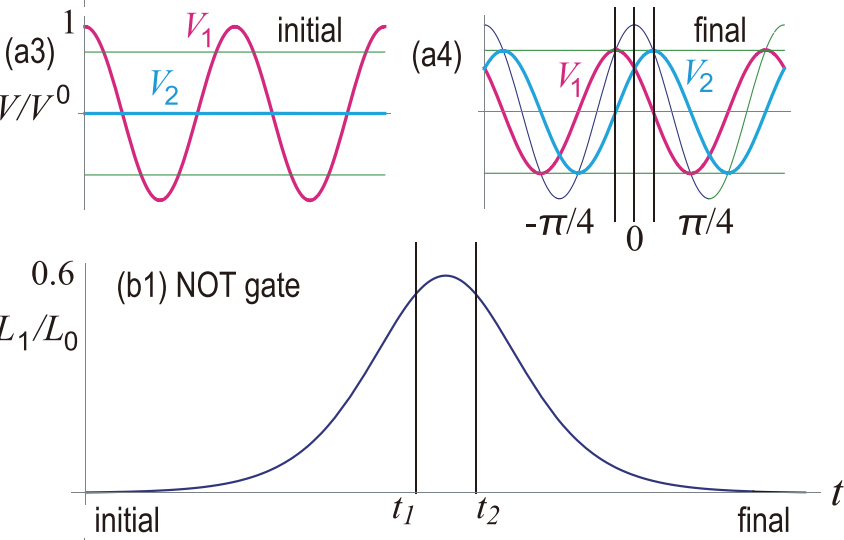

(b2)

$V / V^{0}$
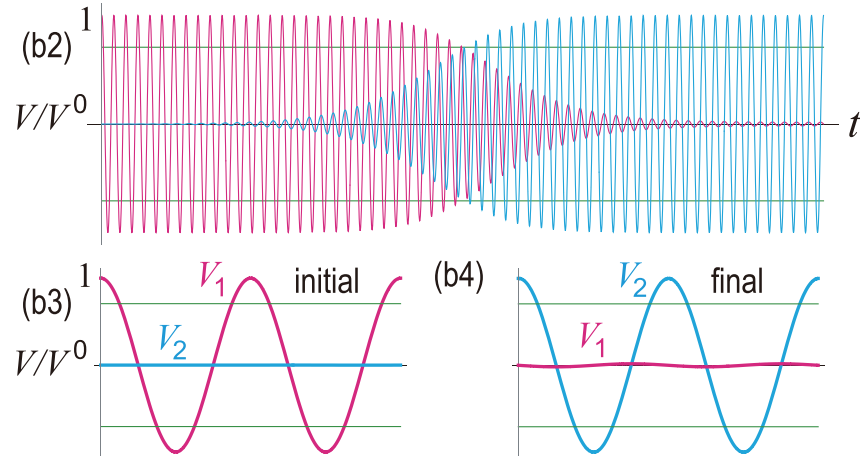

(b4)

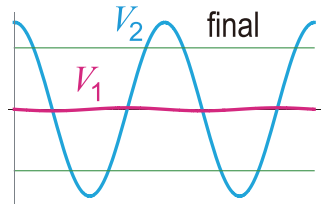

FIG. 3. (a) Mixing gate. (b) NOT gate. [(a1) and (b1)] Timedepending perturbation introduced to the inductance. [(a2) and (b2)] Time evolution of the voltage $V_{1}\left(V_{2}\right)$ at the left (right) $L C$ resonator is represented by a magenta (cyan) curve. (3) Time evolution of $V_{1}$ and $V_{2}$ for $0<\omega_{0} t<4 \pi$, representing the initial state, where $V_{2}=0$. (4) Time evolution $V_{1}$ and $V_{2}$ for $116 \pi<t<120 \pi$, representing the final state, where the voltage without the perturbation is represented by a black curve. We set $L_{1} / L_{0}=0.1$ and $T=10 \omega_{0}$ for $0<\omega_{0} t<$ $120 \pi$. We set $\omega_{0} t_{1}=50 \pi$ and $\omega_{0} t_{2}=55 \pi$ for the mixing gate and $\omega_{0} t_{1}=55 \pi$ and $\omega_{0} t_{2}=65 \pi$ for the NOT gate. The horizontal green lines represent the voltage $\pm V^{0} / \sqrt{2}$.

which we have illustrated in Fig. 3(a).

We solve Eq. (24) numerically with the use of Eq. (29) and show how the voltage evolves in Fig. 3. By tuning $t_{2}-t_{1}$ and $L_{1}$ appropriately, in order to construct the mixing gate (23), we make the magnitudes of $V_{1}$ and $V_{2}$ equal in the final state, i.e., for $t \gg t_{2}$. We find the phase delay $\pi / 4$ in $V_{1}$ and the phase advance $\pi / 4$ in $V_{2}$ as in Fig. 3(a4).

We may discuss the process analytically. For this purpose, we approximate Eq. (29) by a step function such that $1 / L_{1}(t)=0$ for $t<t_{1}, L_{1}(t)=L_{1}$ for $t_{1}<t<t_{2}$ and 
$1 / L_{1}(t)=0$ for $t>t_{2}$. Two resonators are decoupled when $1 / L_{1}(t)=0$. For definiteness, we choose $t_{1}=0$.

First, we analyze the case where only the left $A C$ resonator is active for $t \leqslant 0$, or

$$
V_{1}^{\mathrm{ini}}(t)=V_{0} \cos \left(\omega_{0} t\right), \quad V_{2}^{\mathrm{ini}}(0)=0
$$

At $t=t_{1}$, the perturbation $L_{1}(t)$ is set on.

(i) For $0 \leqslant t \leqslant t_{2}$, we may solve the Kirchhoff equation (24) for the voltages as

$$
\begin{aligned}
& V_{1}(t)=V_{0} \cos \left[\frac{\ell+1}{2} \omega_{0} t\right] \cos \left[\frac{\ell-1}{2} \omega_{0} t\right], \\
& V_{2}(t)=V_{0} \sin \left[\frac{\ell+1}{2} \omega_{0} t\right] \sin \left[\frac{\ell-1}{2} \omega_{0} t\right],
\end{aligned}
$$

where we have chosen the initial condition to meet Eq. (30), or

$$
V_{1}(0)=V_{0}, \quad V_{2}(0)=0 \text {. }
$$

When $\ell \simeq 1$, the oscillation modes are made of the highfrequency mode $\frac{\ell+1}{2} \omega_{0}$ and the low-frequency mode $\frac{\ell-1}{2} \omega_{0}$.

(ii) At $t=t_{2}$, we require the amplitudes of $V_{1}(t)$ and $V_{2}(t)$ to be equal. Since the amplitude is determined by the lowfrequency mode, the condition reads

$$
\cos \left[\frac{\ell-1}{2} \omega_{0} t_{2}\right]=\sin \left[\frac{\ell-1}{2} \omega_{0} t_{2}\right]=\frac{1}{\sqrt{2}} .
$$

Since the connection is weak, we have $L / L_{1} \ll 1$, which leads to $\ell \simeq 1+L / L_{1}$. We use it to derive the relation

$$
\frac{L}{2 L_{1}} \omega_{0} t_{2}=\frac{\pi}{4}
$$

which fixes $t_{2}$ to generate the mixing gate (23). The voltages read

$$
\begin{aligned}
& V_{1}\left(t_{2}\right)=\frac{V_{0}}{\sqrt{2}} \cos \left[\frac{\ell+1}{2} \omega_{0} t_{2}\right]=\frac{V_{0}}{\sqrt{2}} \cos \left[\omega_{0} t_{2}+\frac{\pi}{4}\right], \\
& V_{2}\left(t_{2}\right)=\frac{V_{0}}{\sqrt{2}} \sin \left[\frac{\ell+1}{2} \omega_{0} t_{2}\right]=\frac{V_{0}}{\sqrt{2}} \cos \left[\omega_{0} t_{2}-\frac{\pi}{4}\right],
\end{aligned}
$$

where use was made of Eqs. (31), (32), and (35). There are phase shifts $\pm \frac{\pi}{4}$.

(iii) For $t>t_{2}$, since the perturbation is off, two $L C$ resonators resonate independently with the initial conditions (36) and (37), or

$$
\begin{aligned}
& V_{1}^{\text {fin }}(t)=\frac{V_{0}}{\sqrt{2}} \cos \left[\omega_{0} t+\frac{\pi}{4}\right], \\
& V_{2}^{\text {fin }}(t)=\frac{V_{0}}{\sqrt{2}} \cos \left[\omega_{0} t-\frac{\pi}{4}\right] .
\end{aligned}
$$

It follows that

$$
\begin{gathered}
\alpha_{1}^{\text {ini }}=1, \quad \alpha_{2}^{\text {ini }}=0, \\
\alpha_{1}^{\text {fin }}=\frac{1}{\sqrt{2}} e^{i \pi / 4}, \quad \alpha_{2}^{\text {fin }}=\frac{1}{\sqrt{2}} e^{-i \pi / 4}
\end{gathered}
$$

from Eqs. (4), (30), (38), and (39).

Next, we analyze the case where only the right AC resonator is active for $t \leqslant 0$, or

$$
V_{1}^{\text {ini }}(t)=0, \quad V_{2}^{\text {ini }}(0)=V_{0} \cos \left(\omega_{0} t\right)
$$

instead of Eq. (30): See Fig. 1(c). By making precisely the same analysis, we obtain

$$
\begin{gathered}
\alpha_{1}^{\mathrm{ini}}=0, \quad \alpha_{2}^{\mathrm{ini}}=1 \\
\alpha_{1}^{\mathrm{fin}}=\frac{1}{\sqrt{2}} e^{-i \pi / 4}, \quad \alpha_{2}^{\mathrm{fin}}=\frac{1}{\sqrt{2}} e^{i \pi / 4} .
\end{gathered}
$$

The results (40), (41) (43), and (44) are summarized as the mixing gate (23) based on the definition (6).

NOT gate. The NOT gate is defined by the matrix

$$
U_{\mathrm{NOT}}=\left(\begin{array}{ll}
0 & 1 \\
1 & 0
\end{array}\right)
$$

which acts on one qubit. We find from Eq. (23) that

$$
U_{\mathrm{NOT}}=U_{\mathrm{mix}}^{2} \text {. }
$$

It is given by a sequential application of the mixing gate. The construction is similar to that of the mixing gate provided the duration of the inductor $L_{1}$ is made twice. We present numerical results in Fig. 3(b). With respect to an analytical study, the main equation is

$$
\frac{L}{2 L_{1}} \omega_{0} t_{2}=\frac{\pi}{2}
$$

in place of Eq. (35).

One qubit universal gate. We may construct a combination of the Hadamard and phase-shift gates such as

$$
U_{1 \mathrm{bit}}=e^{-i \theta / 2} U_{\phi+\pi} U_{\mathrm{H}} U_{\theta} U_{\mathrm{H}}=\left(\begin{array}{cc}
\cos \frac{\theta}{2} & -i \sin \frac{\theta}{2} \\
i e^{i \phi} \sin \frac{\theta}{2} & -e^{i \phi} \cos \frac{\theta}{2}
\end{array}\right),
$$

which represents any SU(2) generator. It is called the onequbit universal-quantum gate.

CNOT gate. The CNOT gate is defined by a matrix

$$
U_{\mathrm{CNOT}}=\left(\begin{array}{llll}
1 & 0 & 0 & 0 \\
0 & 1 & 0 & 0 \\
0 & 0 & 0 & 1 \\
0 & 0 & 1 & 0
\end{array}\right),
$$

which acts on the two-qubit state $(|00\rangle,|01\rangle,|10\rangle,|11\rangle)^{t}$. A two-qubit operation is constructed by using four $L C$ resonators as in Fig. 1. The CNOT gate is constructed by applying the NOT gate between the resonators representing $|10\rangle$ and $|11\rangle$, as shown in Fig. 1(d).

Controlled $\mathrm{Z}$ gate. The $\mathrm{CZ}$ gate is defined by a matrix

$$
U_{\mathrm{CZ}}=\operatorname{diag} \cdot[1,1,1,-1],
$$

which acts on the two-qubit state $(|00\rangle,|01\rangle,|10\rangle,|11\rangle)^{t}$. It follows from the definition that the controlled and target 

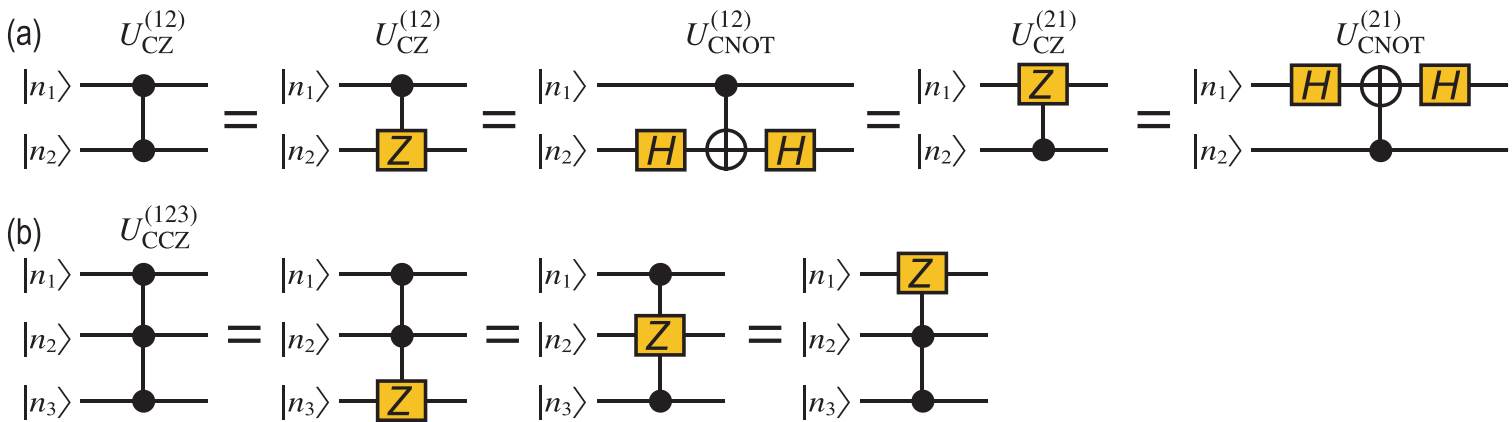

FIG. 4. Equivalent quantum-circuit representation of (a) CZ gate and (b) CCZ gate.

qubits are symmetric in the $\mathrm{CZ}$ gate, which leads to various equivalence quantum circuits as shown in Fig. 4(a). We denote the $\mathrm{CZ}$ gate by the two black disks connected by a line.

$C C Z$ gate. In a similar way to the $\mathrm{CZ}$ gate, we can construct the controlled-controlled $\mathrm{Z}(\mathrm{CCZ})$ gate acting on three qubits. It flips the sign $x_{7}$ of the state $|111\rangle$. Namely, we flip $x_{7}$ to $-x_{7}$. As in the case of the $\mathrm{CZ}$ gate, the $\mathrm{CCZ}$ gate is symmetric with respect to the exchange of the controlled and target qubits shown in Fig. 4(b). We denote the CCZ gate by the three black disks connected by a line.

$C^{p-1} Z$ gate. We further generalize the $\mathrm{CCZ}$ gate to the $\mathrm{C}^{p-1} \mathrm{Z}$ gate. It is a $p$-qubit gate, which flips the sign $x_{2^{p}-1}$ of the coefficient of the state $|11 \cdots 1\rangle$. As in the case of the $\mathrm{CZ}$ and $\mathrm{CCZ}$ gates, the $\mathrm{C}^{p-1} \mathrm{Z}$ gate is symmetric with respect to the controlled and target qubits.

More generally, we may take an $N$-qubit system with $N>p$. We may consider a $\mathrm{C}^{p-1} \mathrm{Z}$ gate acting on a $p$-qubit subspace. We denote it by $p$ black disks connected by a line. Such $C^{p-1} \mathrm{Z}$ gates play an essential role to make a hypergraph state as we will soon see.

Controlled phase-shift gate. The controlled phase-shift gate is defined by the matrix

$$
U_{\mathrm{Z}_{\phi}}=\operatorname{diag} .\left[1,1,1, e^{i \phi}\right],
$$

which acts on two qubits. There is no action on the target qubit if the control qubit is $|0\rangle$, while the $\phi$ phase-shift gate is applied if the control qubit is $|1\rangle$. The controlled phase-shift gate is constructed by applying the phase-shift gate for the $L C$ resonators representing $|11\rangle$, as shown in Fig. 1(e).

Note that the CZ gate (50) is obtained by setting $\phi=\pi$ in the controlled phase-shift gate (51). Namely, Eq. (51) may be viewed as a generalization of the $\mathrm{CZ}$ gate, and hence we call it the $\mathrm{CZ}_{\phi}$ gate.

$C^{p-1} Z_{\phi}$ gate. In a similar way to $\mathrm{C}^{p-1} \mathrm{Z}$ gates, we may define multi-controlled phase-shift gates, which we denote by $\mathrm{C}^{p-1} \mathrm{Z}_{\phi}$ gates. It is a $p$-qubit gate, which multiplies the phase $e^{i \phi}$ to the coefficient $\alpha_{2^{p}-1}$ of the state $|11 \cdots 1\rangle$.

Fidelity. In actual electric circuits, there is some randomness in the values of the capacitance and the inductance, which results in the decrease of the accuracy of the quantum gate. The accuracy of the quantum gates is measured by the fidelity defined by

$$
F\left(\psi^{\prime}, \psi\right) \equiv\left|\left\langle\psi^{\prime} \mid \psi\right\rangle\right|,
$$

where $|\psi\rangle$ is the output wave function from the ideal quantum gate, while $\left|\psi^{\prime}\right\rangle$ is the output wave function from a quantum gate with randomness. The fidelity satisfies the condition,

$$
0 \leqslant F\left(\psi^{\prime}, \psi\right) \leqslant 1,
$$

where $F\left(\psi^{\prime}, \psi\right)=1$ indicates that the quantum gate is ideal.

We introduce randomness into capacitance and inductance uniformly distributing from $-\delta$ to $\delta$ into an electric circuit as

$$
C \longmapsto C(1+\eta \delta), \quad L \longmapsto L(1+\eta \delta),
$$

where $\eta$ is a random variable ranging from -1 to 1 .

Another origin of the decrease of the fidelity is an inaccuracy of the times $t_{1}$ and $t_{2}$ in Eqs. (13) and (29). We introduce an inaccuracy of the time as

$$
C_{1}(t)=\frac{C_{1}^{0}}{2}\left(\tanh \frac{t-\left(t_{1}+\eta_{1}^{t} \delta\right)}{T}-\tanh \frac{t-\left(t_{2}+\eta_{2}^{t} \delta\right)}{T}\right)
$$

and

$$
\frac{1}{L_{1}(t)}=\frac{1}{2 L_{1}}\left(\tanh \frac{t-\left(t_{1}+\eta_{1}^{t} \delta\right)}{T}-\tanh \frac{t-\left(t_{2}+\eta_{2}^{t} \delta\right)}{T}\right),
$$

where $\eta_{1}^{t}, \eta_{2}^{t}$, and $\delta$ are the same as in the case of randomness in the capacitance and the inductance.

We first discuss the fidelity of the phase-shift gate. The phase shift due to the randomness of the capacitances $C$ and $C_{1}$ and the inaccuracy of $t_{1}$ and $t_{2}$ is obtained as

$$
\phi^{\prime}=\frac{C_{1}^{0}\left(1+\eta_{1} \delta\right) \omega_{0}}{2 C(1+\eta \delta)}\left(t_{2}-t_{1}+\left(1+\left(\eta_{2}^{t}-\eta_{1}^{t}\right) \delta\right)\right) .
$$

As far as the inaccuracies $\eta_{1}^{t} \delta$ and $\eta_{2}^{t} \delta$ are concerned, the phase shift depends only on that of the time duration $\left(\eta_{2}^{t}-\eta_{1}^{t}\right) \delta$. The resultant wave function is given by

$$
\left|\psi^{\prime}\right\rangle=\left\{1, e^{i \phi^{\prime}}\right\} / \sqrt{2},
$$

which leads the fidelity $F\left(\psi^{\prime}, \psi\right)=\cos ^{2}\left[\phi-\phi^{\prime}\right]$.

We next calculate the fidelity of the mixing gate by numerically solving Eq. (24). We show the fidelity for the mixing gate as a function of the randomness $\delta$ in Fig. 5(a). It decreases as the increase of the randomness. We show a fidelity with typical randomness in Table I.

TABLE I. Fidelity with typical randomness.

\begin{tabular}{lllll}
\hline \hline randomness in elements & $1 \%$ & $5 \%$ & $10 \%$ & $20 \%$ \\
fidelity $F\left(\psi^{\prime}, \psi\right)$ & 1.000000 & 0.999975 & 0.997186 & 0.996084 \\
\hline
\end{tabular}


(a) randomness in elements

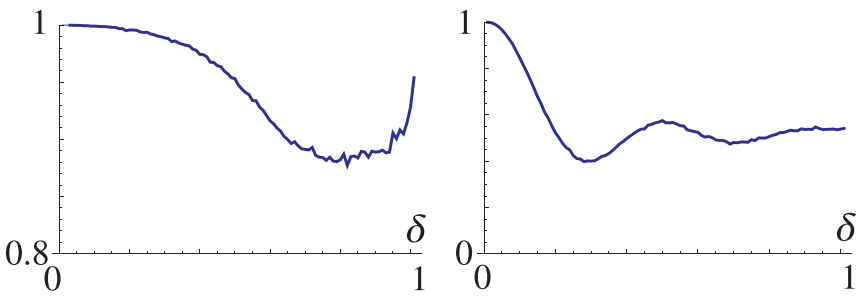

FIG. 5. (a) Mean fidelity as a function of randomness in the capacitance $C$, the inductance $L$ and $L_{1}$. The horizontal axis is the magnitude of randomness $\delta$. (b) Mean fidelity as a function of inaccuracy of $t_{1}$ and $t_{2}$. We have taken 1000 times average. See also the caption of Fig. 3.

We show the fidelity for the mixing gate as a function of the time inaccuracy $\delta$ in Fig. 5(b), which is obtained by numerically solving Eq. (24). We show a fidelity against an inaccuracy of time duration in Table II.

We next analytically study the fidelity of the mixing gate with the time inaccuracy by assuming there is no randomness in the capacitance and the inductance. The voltages due to the time inaccuracy are obtained from Eqs. (31) and (32) as

$$
\begin{aligned}
V_{1}= & V_{0} \cos \left[\frac{\pi}{4}+\frac{L}{2 L_{1}} \omega_{0}\left(\eta_{2}^{t}-\eta_{1}^{t}\right) \delta\right] \\
& \times \cos \left[\omega_{0} t_{2}+\frac{\pi}{4}+\frac{L}{2 L_{1}} \omega_{0}\left(\eta_{2}^{t}-\eta_{1}^{t}\right) \delta\right], \\
V_{2}= & V_{0} \sin \left[\frac{\pi}{4}+\frac{L}{2 L_{1}} \omega_{0}\left(\eta_{2}^{t}-\eta_{1}^{t}\right) \delta\right] \\
& \times \cos \left[\omega_{0} t_{2}-\frac{\pi}{4}+\frac{L}{2 L_{1}} \omega_{0}\left(\eta_{2}^{t}-\eta_{1}^{t}\right) \delta\right],
\end{aligned}
$$

It corresponds to the output wave function

$$
\begin{aligned}
& \alpha_{1}^{\mathrm{fin}}=\cos \left[\frac{\pi}{4}+\frac{L}{2 L_{1}} \omega_{0}\left(\eta_{2}^{t}-\eta_{1}^{t}\right) \delta\right] e^{i\left(\frac{\pi}{4}+\frac{L}{2 L_{1}} \omega_{0}\left(\eta_{2}^{t}-\eta_{1}^{t}\right) \delta\right)}, \\
& \alpha_{2}^{\mathrm{fin}}=\sin \left[\frac{\pi}{4}+\frac{L}{2 L_{1}} \omega_{0}\left(\eta_{2}^{t}-\eta_{1}^{t}\right) \delta\right] e^{i\left(-\frac{\pi}{4}+\frac{L}{2 L_{1}} \omega_{0}\left(\eta_{2}^{t}-\eta_{1}^{t}\right) \delta\right)} .
\end{aligned}
$$

The fidelity is calculated as

$$
F\left(\psi^{\prime}, \psi\right)=\cos \left[\frac{L}{2 L_{1}} \omega_{0}\left(\eta_{2}^{t}-\eta_{1}^{t}\right) \delta\right] .
$$

It explains an oscillatory behavior in Fig. 5(b).

\section{ARTIFICIAL NEURON}

An artificial neuron is a mathematical model $[43,44]$ to simulate a biological neuron. There are $m$ inputs $x_{0}, x_{1}, \cdots$, $x_{m-1}$ and $m$ weights $w_{0}, w_{1}, \cdots, w_{m-1}$, where $x_{j}$ and $w_{j}$ are real numbers. We represent the input and the weight by wave

TABLE II. Fidelity with typical inaccuracy of time duration.

\begin{tabular}{lllll}
\hline \hline inaccuracy of time & $50 \pm 0.01$ & $50 \pm 0.05$ & $50 \pm 0.1$ & $50 \pm 0.2$ \\
fidelity $F\left(\psi^{\prime}, \psi\right)$ & 0.997856 & 0.948688 & 0.809312 & 0.50156 \\
\hline \hline
\end{tabular}

(a) artificial neuron

inputs weights

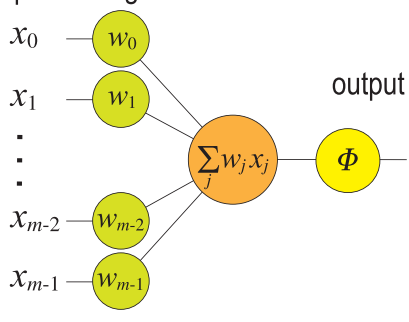

(b) complex neuron inputs weights

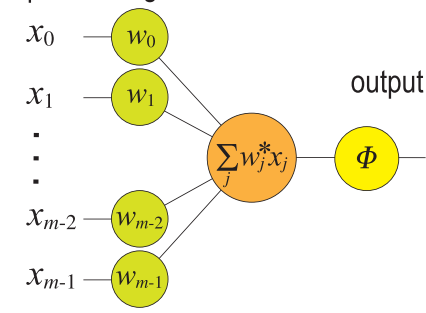

FIG. 6. (a) Schematic for an artificial neuron. It has $m$ real inputs $x_{j}$ and real internal degree of freedom named weights $w_{j}$. Output is obtained by calculating the inner product $\sum_{j} w_{j} x_{j}$ and then by applying an activation function $\Phi\left(\sum_{j} w_{j} x_{j}\right)$. (b) Schematic for a complex neuron. It has complex $m$ inputs $x_{j}$ and complex weights $w_{j}$ with the inner product $\sum_{j} w_{j}^{*} x_{j}$.

functions as [48]

$$
\left.\left.\left|\psi_{x}\right\rangle=\frac{1}{\sqrt{2^{N}}} \sum_{j=0}^{2^{N}-1} x_{j}|j\rangle\right\rangle, \quad\left|\psi_{w}\right\rangle=\frac{1}{\sqrt{2^{N}}} \sum_{j=0}^{2^{N}-1} w_{j}|j\rangle\right\rangle,
$$

where $|j\rangle\rangle$ forms the $N$ qubit basis as in Eq. (3). Note the difference between the coefficients $\alpha_{j}$ in Eq. (3) and $x_{j}, w_{j}$ in Eq. (64) by the factor $1 / \sqrt{2^{N}}$.

The first step in the artificial neuron is to calculate the inner product $\sum_{j} w_{j} x_{j}$. The inner product of the input data and the weight data measures the similarity between them. For instance, using a series of numbers representing a set of reference patterns as the weight, we may calculate the similarity between an input pattern and the reference pattern.

The inner product is outputted after applying an activation function,

$$
y=\Phi\left(\sum_{j} w_{j} x_{j}\right) .
$$

The activation function $\Phi$ has various forms such as the step function [56,57], a linear function, a sigmoid function, a ramp function [58] and so on. We show a schematic of a neuron in Fig. 6(a). In the process of artificial neuron, the heaviest procedure is the calculation of $\sum_{j} w_{j} x_{j}$, which is efficiently done by using a quantum computer [48].

We implement the wave functions (64) by unitary transformations from the initial state $|0\rangle\rangle$,

$$
\left.\left.\left|\psi_{x}\right\rangle=U_{x}|0\rangle\right\rangle, \quad\left|\psi_{w}\right\rangle=U_{w}^{\dagger}|0\rangle\right\rangle .
$$

Then, the inner product is calculated as

$$
\sum_{j} w_{j} x_{j}=2^{N}\left\langle\psi_{w} \mid \psi_{x}\right\rangle=2^{N}\left\langle\left\langle 0\left|U_{w} U_{x}\right| 0\right\rangle\right\rangle .
$$

We explicitly construct $U_{x}$ and $U_{w}$ later in this section. On the other hand, the application of the activation function $\Phi$ is easy with the use of a classical computer since it is a one-to-one map.

A simplest artificial neuron is given by the perceptron model $[56,57]$. Here, the input and the weight wave functions are given by Eq. (64) with $x_{j}= \pm 1$ and $w_{j}= \pm 1$. Such states are called real equally weighted (REW) states. Furthermore, 
the step function is used as the activation function,

$$
y=\Theta\left(\sum_{j} w_{j} x_{j}-h\right),
$$

where $\Theta$ is a step function with the threshold $h, \Theta(x-h)=1$ for $x \geqslant h$ and $\Theta(x-h)=-1$ for $x<h$.

In our application of artificial neuron to pattern recognition we use REW states as in the perceptron model but without employing the activation function (68). We use the inner product itself as the output.

We now discuss how to construct a REW state from the initial state $|0\rangle\rangle$, or how to determine $U_{x}$ and $U_{w}^{\dagger}$ in Eq. (66) in the standard quantum-circuit implementation [48] and also in the electric-circuit implementation.

In the first step, we prepare the equal-coefficient state defined by

$$
\left.\left|\psi_{0}\right\rangle=\frac{1}{\sqrt{2^{N}}} \sum_{j=0}^{2^{N}-1}|j\rangle\right\rangle \equiv \frac{1}{\sqrt{2^{N}}} \sum_{n_{j}=0,1}\left|n_{1} n_{2} \cdots n_{N}\right\rangle .
$$

This is done by way of the Walsh-Hadamard transform of the initial state $|0\rangle\rangle$,

$$
\left.\left|\psi_{0}\right\rangle=\bigotimes_{s=1}^{N} U_{\mathrm{H}}^{(s)}|0\rangle\right\rangle,
$$

where $U_{\mathrm{H}}^{(s)}$ is the Hadamard gate acting on the $s$ th qubit.

In the second step, we construct $\left|\psi_{x}\right\rangle$ and $\left|\psi_{w}\right\rangle$ from the equal-coefficient state as

$$
\left|\psi_{x}\right\rangle=V_{x}\left|\psi_{0}\right\rangle, \quad\left|\psi_{w}\right\rangle=V_{w}^{\dagger}\left|\psi_{0}\right\rangle .
$$

Here, $V_{x}$ is an operation by changing the coefficient $x_{j}$ in the state $\left|\psi_{x}\right\rangle$ to $-x_{j}$ if $x_{j}=-1$ for all $j$. Hence, $V_{x}$ is given by a sequential application of $\mathrm{C}^{p-1} \mathrm{Z}$ gates. For this purpose, we search for the qubit state $|j\rangle\rangle$ whose coefficient is $x_{j}=-1$. Then, we apply an appropriate $\mathrm{C}^{p-1} \mathrm{Z}$ gate to the state to change its coefficient to $x_{j}=1$. An explicit example is given in Appendix A.

We find from Eqs. (66), (70), and (71) that

$$
U_{x}=V_{x} \bigotimes_{s=1}^{N} U_{\mathrm{H}}^{(s)}, \quad U_{w}^{\dagger}=V_{w}^{\dagger} \bigotimes_{s=1}^{N} U_{\mathrm{H}}^{(s)},
$$

and from Eqs. (67) and (72) that

$$
\sum_{j} w_{j} x_{j}=2^{N}\left\langle\psi_{w} \mid \psi_{x}\right\rangle=2^{N}\left\langle\left\langle 0\left|\bigotimes_{s=1}^{N} U_{\mathrm{H}}^{(s)} V_{w} V_{x} \bigotimes_{s=1}^{N} U_{\mathrm{H}}^{(s)}\right| 0\right\rangle\right\rangle .
$$

This is the basic formula to calculate the inner product by a quantum computer starting from the initial state $|0\rangle\rangle$. An explicit example of implementation is given in Sec. VII.

\section{PATTERN RECOGNITION}

Pattern recognition is one of the most useful applications of artificial neurons. As an example, we consider a pattern made of rectangular pixels painted in black and white. We show two patterns made of $5 \times 4$ pixels and $6 \times 5$ pixels, which are labeled by binary codes as in Figs. 7(a) and 7(b). (a) $5 \times 4$ pixels

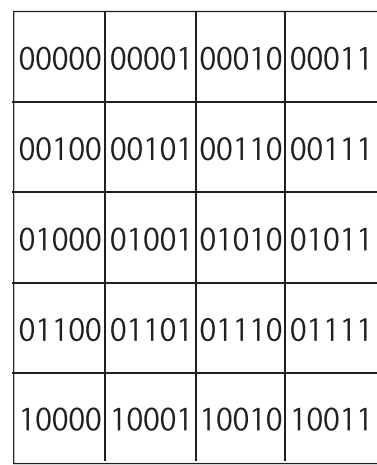
number recognition and (b) $6 \times 5$ pixels for the alphabet recognition.

Next, we assign $x_{j}=1$ for white pixel and $x_{j}=-1$ for black pixel. Here, $j$ is a decimal number representing a binary code assigned to a pixel, $0 \leqslant j \leqslant N_{p}-1$.

In order to represent $N_{x} \times N_{y}$ pixels, we prepare $N$ qubits satisfying $2^{N-1}<N_{x} \times N_{y} \leqslant 2^{N}$. These $N$-qubit states are REW states, which are Eq. (64) with $x_{j}= \pm 1$ and $w_{j}= \pm 1$. Let there be $N_{p}$ patterns to be classified. For instance, it is $N_{p}=10$ for the number recognition and $N_{p}=26$ for the alphabet recognition as in Figs. 8(a) and 8(b), respectively. We use a set of reference patterns as the weight wave function $\left|\psi_{w}(j)\right\rangle$, and compare them with a set of input patterns $\left|\psi_{x}(j)\right\rangle$ : examples are given in Fig. 9 for $N_{p}=10$ and in Fig. 10 for $N_{p}=26$. In these cases, it is enough to prepare five qubits.

We estimate the similarity between an input pattern and the reference pattern by calculating the inner product $\left\langle\psi_{w} \mid \psi_{x}\right\rangle$. We determine which input pattern is most similar to the reference pattern by searching the largest inner product $\left\langle\psi_{w} \mid \psi_{x}\right\rangle$.

(a)
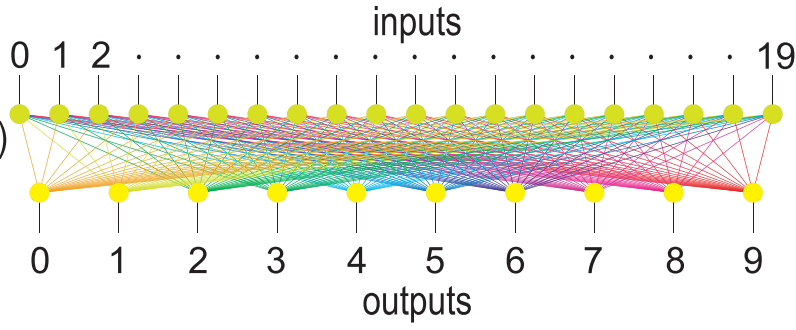

inputs

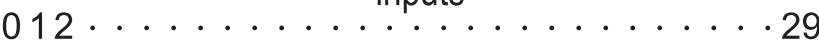

(b)

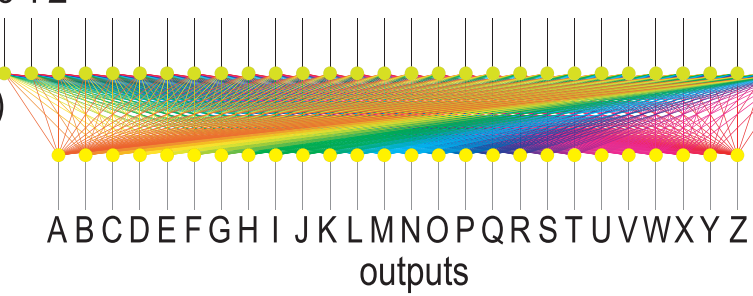

FIG. 8. Neural networks for (a) number recognition and (b) alphabet recognition. 
(a)

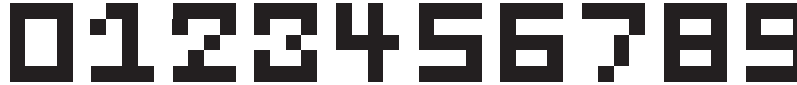
(b)

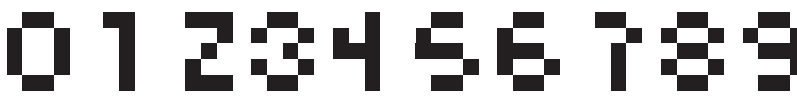

(c)

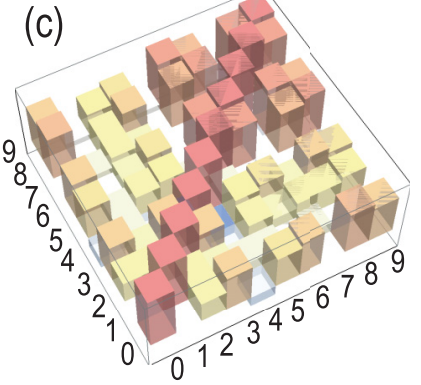

(d)

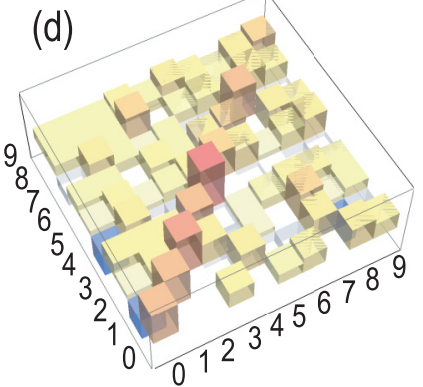

FIG. 9. (a) Reference patterns and (b) input patterns of numbers. (c) Self similarity $\left\langle\psi_{w} \mid \psi_{w}\right\rangle$ and (d) cross similarity $\left\langle\psi_{w} \mid \psi_{x}\right\rangle$ of the number recognition.

This process is expressed by a single layer neural network with $N_{x} \times N_{y}$ inputs and $N_{p}$ outputs as in Fig. 8 . The inner product is calculated as

$$
\left\langle\psi_{w} \mid \psi_{x}\right\rangle=\frac{N_{p}-2 N_{\text {error }}}{N_{p}},
$$

where $N_{\text {error }}$ is the number of errors between the reference and the input patterns defined by

$$
N_{\text {error }}=\sum_{j=0}^{N_{p}-1}\left(\frac{x_{j}-w_{j}}{2}\right)^{2}
$$

We note that $\left\langle\psi_{w} \mid \psi_{x}\right\rangle$ can be negative for $N_{\text {error }} \geqslant N_{p} / 2$. We find $\left|\left\langle\psi_{w} \mid \psi_{x}\right\rangle\right| \leqslant 1$, where $\left\langle\psi_{w} \mid \psi_{x}\right\rangle=1$ indicates the perfect matching.
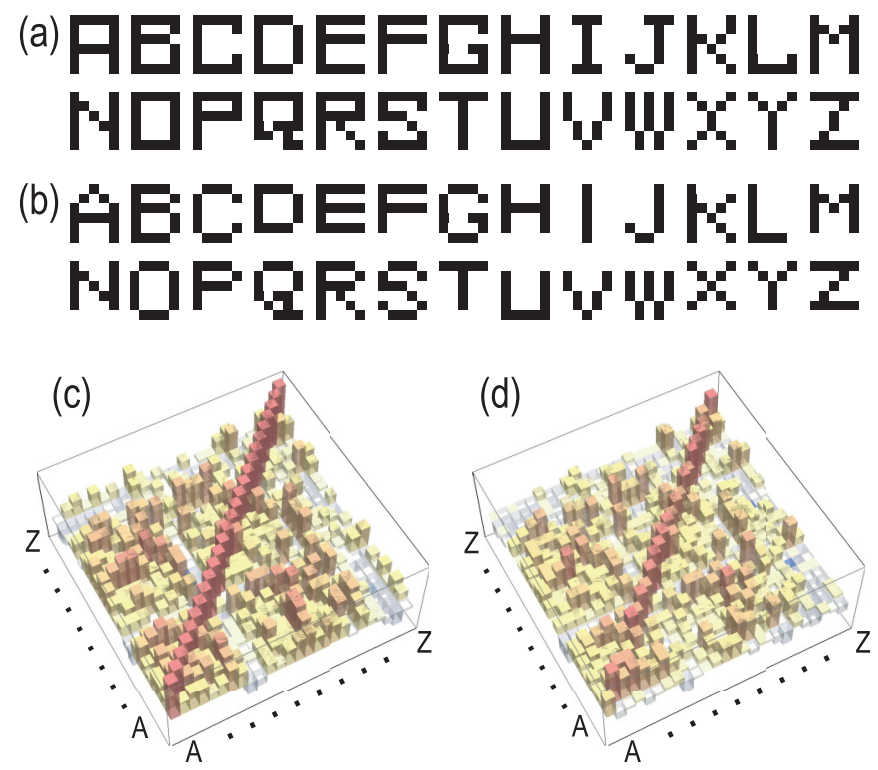

FIG. 10. (a) Reference patterns and (b) input patterns of alphabets. (c) Self similarity $\left\langle\psi_{w} \mid \psi_{w}\right\rangle$ and (d) cross similarity $\left\langle\psi_{w} \mid \psi_{x}\right\rangle$ of the alphabet recognition.
As the first example, we study a recognition of numbers. We choose a set of the reference patterns of numbers as given by Fig. 9(a). We implement them into a wave function $\left|\psi_{w}\right\rangle$. Explicit forms are shown in Appendix B. Then, we take a set of input patterns. See Fig. 9(b) for an instance. First, we calculate the self similarity defined by $\left\langle\psi_{w}\left(j_{1}\right) \mid \psi_{w}\left(j_{2}\right)\right\rangle$, which is shown in Fig. 9(c). The maximum values are taken when $j=j_{1}=j_{2}$ with $\left\langle\psi_{w}(j) \mid \psi_{w}(j)\right\rangle=1$. In order to well recognize different patterns as different ones, it is necessary that $\left\langle\psi_{w}\left(j_{1}\right) \mid \psi_{w}\left(j_{2}\right)\right\rangle$ is small for $j_{1} \neq j_{2}$. From Fig. 9(c), we find that $1,2,3,4,5$, and 7 are well distinguishable because $\left\langle\psi_{w}\left(j_{1}\right) \mid \psi_{w}\left(j_{2}\right)\right\rangle$ is low. On the other hand, 6, 8, and 9 are hardly distinguishable because the similarity is 0.9 , where only one pixel is different.

Next, we study a cross similarity between the input and the reference patterns by calculating $\left\langle\psi_{w}\left(j_{1}\right) \mid \psi_{x}\left(j_{2}\right)\right\rangle$. We fix $j_{2}$ for the input pattern and determine which reference pattern is most similar by choosing the largest inner product. We find 0 , $1,2,3,4,5,6,7$, and 9 are correctly recognized, but 8 is ill recognized to be 3. See Fig. 9(d).

In a similar way, we study an alphabet recognition. We choose a set of the reference patterns of alphabets as in Fig. 10(a) and a set of input patterns in Fig. 10(b). The selfsimilarity and the cross-similarity are shown in Figs. 10(c) and 10(d). We find the following properties from the self similarity: Alphabets are easier to differentiate comparing to numbers. " $F$ " is hardly differentiated from "P", where the similarity is $14 / 15$. "C", "D", "G," and "O" are hardly differentiable among themselves, and " $\mathrm{M}$ " and " $\mathrm{N}$ " are hardly differentiated one another, where the similarity is $13 / 15$. We find the following properties from the cross similarity: There are ill recognitions of "D" to "Q", "E" to "F", "O" to " $D$ ", "X" to "Y" and " $\mathrm{Z}$ " to "T". In addition, "C" has equal similarity to both "C" and " $\mathrm{D}$ " in the reference pattern, "G" has equal similarity to both "D" and "G". "I" has equal similarity to both "I" and "T". For other cases, the input patterns are well recognized with respect to the reference patterns.

\section{COMPLEX-ARTIFICIAL NEURON}

We proceed to study a complex-artificial neuron, where the input and the weight are given by CEW states. Namely, the wave functions are given by Eq. (64) with complex coefficients $x_{j}=e^{i \theta_{j}^{x}}$ and $w_{j}=e^{i \theta_{j}^{w}}$. The inner product reads

$$
\left\langle\psi_{w} \mid \psi_{x}\right\rangle=\frac{1}{2^{N}} \sum_{j} w_{j}^{*} x_{j}=\frac{1}{2^{N}} \sum_{j} e^{i\left(\theta_{j}^{*}-\theta_{j}^{w}\right)} .
$$

The output is given by [52]

$$
y=\Phi\left(\sum_{j} w_{j}^{*} x_{j}\right),
$$

where $\Phi$ is a complex-activation function.

Any CEW state is generated by a sequential application of $\mathrm{C}^{p-1} \mathrm{Z}_{\phi}$ gates to the equal-coefficient state (69) precisely as the REW state is generated by a sequential application of $\mathrm{C}^{p-1} \mathrm{Z}$ gates to it. Let us explain it by taking the most general 

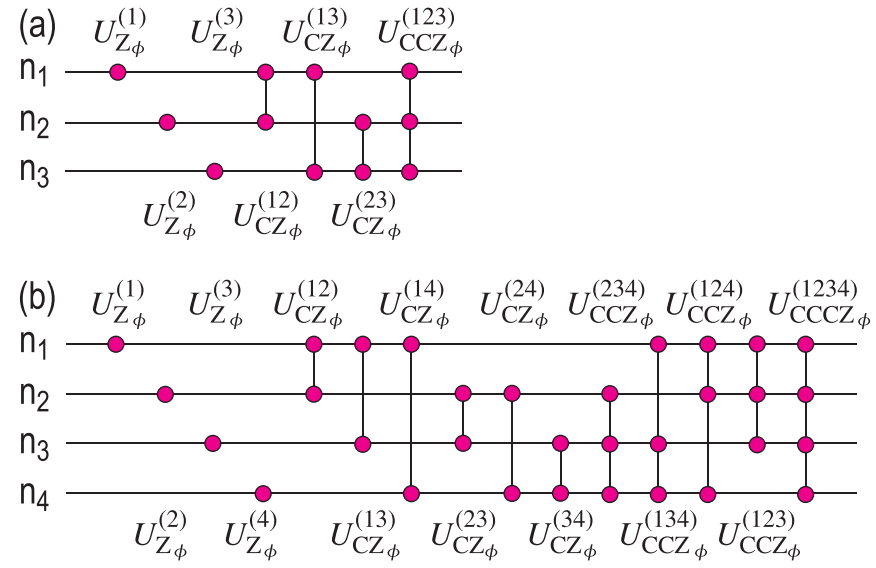

FIG. 11. Standard quantum circuits for a generation process of the CEW state for (a) three qubits and (b) four qubits. An isolated magenta disk indicates a $\mathrm{Z}$ gate. Magenta disks connected by a line indicate a $\mathrm{C}^{p-1} \mathrm{Z}_{\phi}$ gate.

CEW state $|\psi\rangle$ in the three-qubit system. It is given by

$$
|\psi\rangle=\sum_{n_{j}=0,1} \alpha_{n_{1} n_{2} n_{3}}\left|n_{1} n_{2} n_{3}\right\rangle,
$$

with

$$
\alpha_{n_{1} n_{2} n_{3}}=\frac{1}{\sqrt{2^{N}}} \exp \left(i \theta_{n_{1} n_{2} n_{3}}\right),
$$

where we set $\theta_{000}=0$ without loss of generality.

We list up all possible $\mathrm{C}^{p-1} \mathrm{Z}_{\phi}$ gates in Fig. 11(a). Recall that all $\mathrm{C}^{p-1} \mathrm{Z}_{\phi}$ gates are commutative. The generated CEW state is given by

$$
\begin{aligned}
\left.U_{\mathrm{CCZ}_{\phi_{123}}}^{(123)} U_{\mathrm{CZ}_{\phi_{23}}}^{(23)} U_{\mathrm{CZ}_{\phi_{13}}^{(13)}}^{(12)} U_{\mathrm{CZ}_{\phi_{12}}}^{(12)} U_{\mathrm{Z}_{\phi_{3}}}^{(3)} U_{\mathrm{Z}_{\phi_{2}}}^{(2)} U_{\mathrm{Z}_{\phi_{1}}}^{(1)} \bigotimes_{s=1}^{4} U_{\mathrm{H}}^{(s)}|0\rangle\right\rangle \\
=\frac{1}{\sqrt{8}}\left(|000\rangle+e^{i \phi_{3}}|001\rangle+e^{i \phi_{2}}|010\rangle+e^{i\left(\phi_{2}+\phi_{3}+\phi_{23}\right)}|011\rangle\right. \\
\quad+e^{i \phi_{1}}|100\rangle+e^{i\left(\phi_{1}+\phi_{3}+\phi_{13}\right)}|101\rangle+e^{i\left(\phi_{1}+\phi_{2}+\phi_{12}\right)}|110\rangle \\
\left.\quad+e^{i\left(\phi_{1}+\phi_{2}+\phi_{3}+\phi_{12}+\phi_{13}+\phi_{23}+\phi_{123}\right)}|111\rangle\right)
\end{aligned}
$$

where the angle $\phi_{1}$ is that of the $\mathrm{Z}_{\phi_{1}}$ gate, $\phi_{12}$ is that of $\mathrm{CZ}_{\phi_{12}}$ and $\phi_{123}$ is that of $\mathrm{CCZ}_{\phi_{123}}$, and so on.

It is easy to see that the angles associated with $\mathrm{C}^{p-1} \mathrm{Z}_{\phi}$ gates (80) are uniquely fixed in terms of $\theta_{n_{1} n_{2} n_{3}}$ in the given CEW state (78) because there are seven independent variables in both of these equations. Indeed, by equating Eqs. (78) and (80), we obtain relations

$$
\begin{aligned}
\phi_{1} & =\theta_{100}, \quad \phi_{2}=\theta_{010}, \quad \phi_{3}=\theta_{001}, \\
\phi_{12} & =\theta_{110}-\theta_{100}-\theta_{010}, \\
\phi_{13} & =\theta_{101}-\theta_{100}-\theta_{001}, \\
\phi_{23} & =\theta_{011}-\theta_{010}-\theta_{001}, \\
\phi_{123} & =\theta_{111}+\theta_{100}+\theta_{010}+\theta_{001}-\theta_{110}-\theta_{101}-\theta_{011} .
\end{aligned}
$$
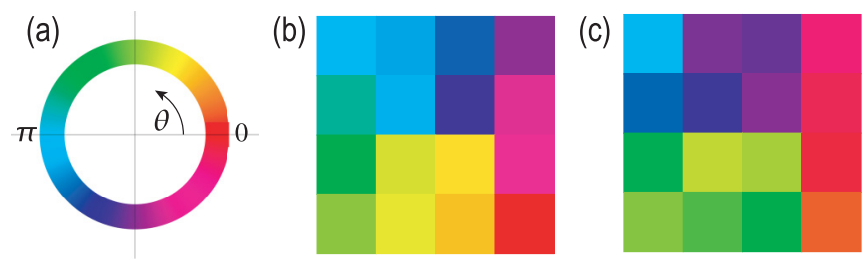

FIG. 12. (a) Color circle, (b) reference color pattern and (c) input color pattern. The input pattern is made by changing randomly colors of the reference pattern within $20 \%$ randomnes.

We have shown which $\mathrm{C}^{p-1} \mathrm{Z}_{\phi}$ gates we have to prepare in order to generate the most general CEW state (78) in the threequbit system.

We also list up all possible $\mathrm{C}^{p-1} \mathrm{Z}_{\phi}$ gates for the 4-qubit system in Fig. 11(b). In general, we can always construct an arbitrary CEW state by applying $\mathrm{C}^{p-1} \mathrm{Z}_{\phi}$ gates in $N$-qubit systems.

\section{COLORED PATTERN RECOGNITION}

The color circle is a color pallet indexed by a number on a circle as shown in Fig. 12(a). It has a one-to-one correspondence to $e^{i \theta}$. For example, $\theta=0$ indicates red and $\theta=\pi$ indicates cyan. Hence, a color pattern made of pixels is well represented by a CEW state. By using a complex neural network, we can estimate a similarity between two colored patterns.

The merit of our color representation scheme is that the color circle is naturally represented by a continuous circle $e^{i \theta}$. In the standard digital representation, we have to digitalize color. The number of classical bits increases as the increase of hue decomposition. On the other hand, all color is continuously represented by one number $e^{i \theta}$ in our scheme.

For example, we show a reference colored pattern in Fig. 12(b). It is enough to prepare 4 qubits for a pattern with 16 pixels. We make an input colored pattern by modifying color randomly within $20 \%$. The inner product of two patterns is $0.993-0.096 i$. The similarity is relatively large although the color of each pixel is modified by $20 \%$. This is because the input pattern is created from the reference pattern by adding noise, where the noise is canceled by adding all contributions from 16 pixels. Hence, our scheme can evaluate similarity between two colored patterns with color noise.

\section{ELECTRIC-CIRCUIT IMPLEMENTATION}

We implement these models by a set of $L C$ resonators. We prepare $2^{N} L C$ resonators to represent the states $\left.|j\rangle\right\rangle$ or $\left|n_{1} n_{2} \cdots n_{s} \cdots n_{N}\right\rangle$. The main issue is the electric-circuit implementation of the inner product formula (73), or

$$
\left\langle\psi_{w} \mid \psi_{x}\right\rangle=\left\langle\left\langle 0\left|\bigotimes_{s=1}^{N} U_{\mathrm{H}}^{(s)} V_{w} V_{x} \bigotimes_{s=1}^{N} U_{\mathrm{H}}^{(s)}\right| 0\right\rangle\right\rangle,
$$

which may be used for CEW states as well as REW states.

The first step is the construction of the equal-coefficient state (69) by applying $\bigotimes_{s=1}^{N} U_{\mathrm{H}}^{(s)}$ to the initial state $\left.|0\rangle\right\rangle$. The action of the Hadamard transformation $U_{\mathrm{H}}^{(s)}$ for the sth qubit 


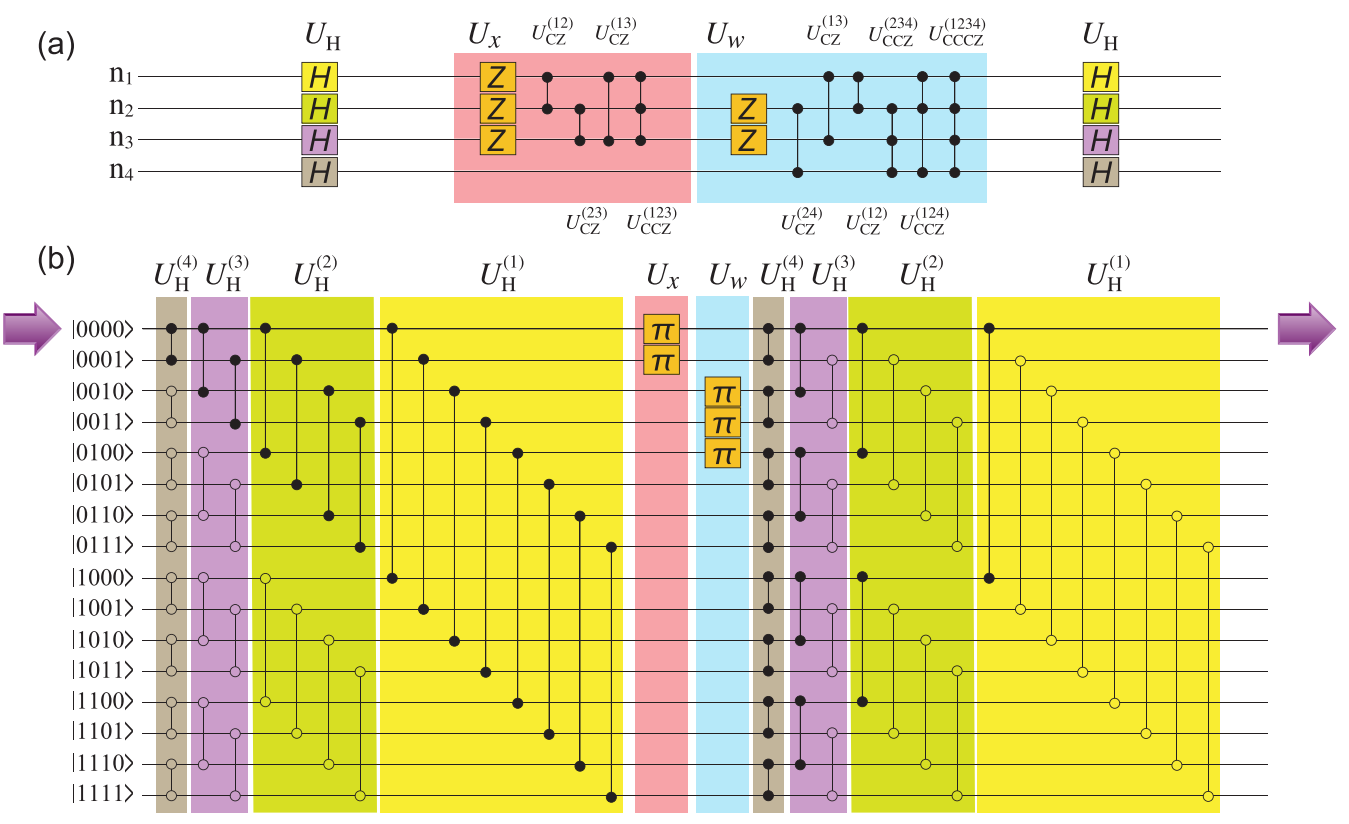

FIG. 13. (a) Standard quantum-circuit model for the calculation of the inner product (82) for an example given by (85) and (86). We use a four-qubit state $\left|n_{1} n_{2} n_{3} n_{4}\right\rangle$ (b) Corresponding electric-circuit simulation. The Hadamard gates are denoted by filled or unfilled black disks connected by a link. The Hadamard bridges with unfilled disks are not necessary since they are irrelevant for the input state $|0\rangle\rangle$ and the output state $\langle\langle 0|$.

is simulated by bridging two resonators $\left|n_{1} n_{2} \cdots n_{s} \cdots n_{N}\right\rangle$ and $\left|n_{1} n_{2} \cdots \bar{n}_{s} \cdots n_{N}\right\rangle$, when $\bar{n}_{s}=1$ for $n_{s}=0$ and $\bar{n}_{s}=0$ for $n_{s}=1$. In the case of $N=4$, the Hadamard gate $U_{\mathrm{H}}^{(1)}$ is simulated by the eight bridges between

$|0000\rangle$ and $|1000\rangle$,
$|0001\rangle$ and $|1001\rangle$,
$|0010\rangle$ and $|1010\rangle$,
$|0011\rangle$ and $|1011\rangle$,
$|0100\rangle$ and $|1100\rangle$,
$|0101\rangle$ and $|1101\rangle$,
$|0110\rangle$ and $|1110\rangle$,
$|0111\rangle$ and $|1111\rangle$.

Although there are many bridges, their assignment is systematic. Apparently we need $N 2^{N-1}$ operations. Actually, many bridges shown by unfilled disks in Fig. 13(b) are not necessary since we start with $|0\rangle\rangle$ and end up with $\langle\langle 0|$ as in Eq. (82). Then, we may delete all operations which is irrelevant to the input and the output, which greatly reduces the number of operations. The necessary operation relating to the $N$-qubit Hadamard gate is $\sum_{s}^{N} 2^{s-1}=2^{N}-1$. The reduction rate is

$$
\lim _{N \rightarrow \infty} \frac{2^{N}-1}{N 2^{N-1}}=\lim _{N \rightarrow \infty} \frac{2}{N} .
$$

The second step is the operation of $\mathrm{C}^{p-1} \mathrm{Z}$ gates in the case of REW states. In construct to the application of $\mathrm{C}^{p-1} \mathrm{Z}$ gates in the standard quantum-circuit implementation, it is enough to apply the $\pi$ phase-shift only for $|j\rangle\rangle$ with $x_{j}=-1$. More explicitly, the CZ gate for two qubits is simulated by the $\pi$ phase-shift gate applied to the resonator representing $|11\rangle$, while the CCZ gate for three qubits is simulated by the $\pi$ phase-shift gate applied to the resonator representing $|111\rangle$. In general, the $\mathrm{C}^{p-1} \mathrm{Z}$ gate for $p$ qubits is simulated by the $\pi$ phase-shift gate applied only to the resonator representing $|11 \cdots 1\rangle$.

We consider an example of an inner product for the input and the weight states given by

$$
\begin{aligned}
4\left|\psi_{x}\right\rangle= & -|0000\rangle-|0001\rangle+|0010\rangle+|0011\rangle \\
& +|0100\rangle+|0101\rangle+|0110\rangle+|0111\rangle \\
& +|1000\rangle+|1001\rangle+|1010\rangle+|1011\rangle \\
& +|1100\rangle+|1101\rangle+|1110\rangle+|1111\rangle
\end{aligned}
$$

and

$$
\begin{aligned}
4\left|\psi_{w}\right\rangle= & |0000\rangle+|0001\rangle-|0010\rangle-|0011\rangle \\
& -|0100\rangle+|0101\rangle+|0110\rangle+|0111\rangle \\
& +|1000\rangle+|1001\rangle+|1010\rangle+|1011\rangle \\
& +|1100\rangle+|1101\rangle+|1110\rangle+|1111\rangle,
\end{aligned}
$$

where the inner product is $\left\langle\psi_{w} \mid \psi_{x}\right\rangle=3 / 8$. The quantum circuit and the electric circuit to calculate this inner product based on the inner product formula (73) are given in Fig. 13(a). Then, we apply the $\pi$ phase-shift gate for $|0000\rangle$ and $|0001\rangle$ in order to construct $\left|\psi_{x}\right\rangle$, while we apply the $\pi$ phase-shift gate for $|0010\rangle,|0011\rangle$ and $|0100\rangle$ in order to construct $\left|\psi_{w}\right\rangle$ as in Fig. 13(b).

It is convenient to define the output $y_{j}$ for each resonator representing $|j\rangle\rangle$ in Fig. 14 by

$$
\left.\frac{1}{4} \sum_{j=0}^{15} y_{j}|j\rangle\right\rangle=\left(\bigotimes_{s=1}^{4} U_{\mathrm{H}}^{(s)}\right) V_{w} V_{x}\left(\bigotimes_{s=1}^{4} U_{\mathrm{H}}^{(s)}\right)|0000\rangle .
$$




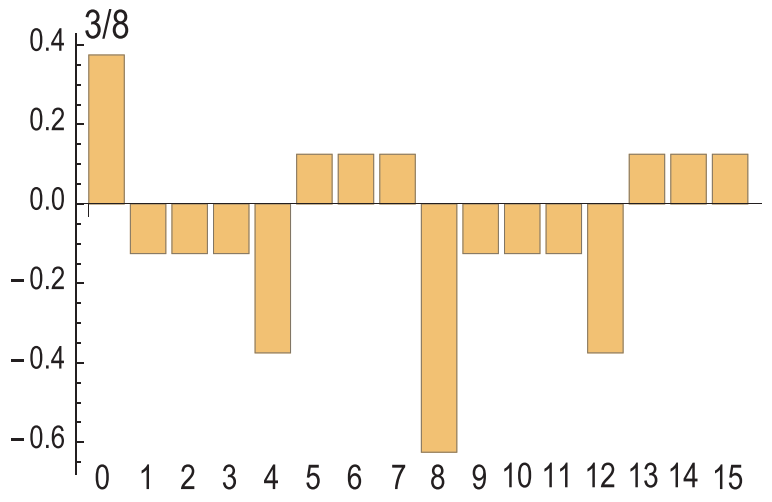

FIG. 14. Output $y_{j}$ for each resonator representing $\left.|j\rangle\right\rangle$. We measure the output $y_{0}$ for the state $\left.|0\rangle\right\rangle$, which gives an inner product $3 / 8=0.375$.

The outs $y_{j}$ are always real. Especially, we are interested in the output for $|0000\rangle$, which is $\mathrm{y}_{0}=3 / 8$. It reproduce a correct the inner product given below Eq. (86).

The generalization to the calculation of an inner product of CEW states is straightforward. As a characteristic feature of the present electric-circuit simulation, it is possible to create a $\phi$ phase-shift gate with an arbitrary angle $\phi$ just by tuning the capacitance of the relevant $L C$ resonator according the formula (19). Hence, any CEW state is generated by applying $\mathrm{C}^{p-1} \mathrm{Z}_{\phi}$ gates with the use of appropriate $\phi$ phase-shift gates.

\section{GRAPH THEORY}

Graph states and hypergraph states. It is intriguing to recapture the REW state in the context of graph theory. Such a state is referred to as a graph state [59-61] that is constructed by a sequential application of $\mathrm{Z}$ gates and $\mathrm{CZ}$ gates to the equal-coefficient state (69). The order of a $\mathrm{Z}$ gate and a $\mathrm{CZ}$ gate is irrelevant because they are diagonal operators and commutable. Then, we may establish one-to-one correspondence between a graph and a graph state. Indeed, in order to make a graph corresponding to a graph state, we first prepare $N$ vertices representing $N$ qubits, as in Fig. 15(a2) for an instance of $N=4$. We add self-loop links to the vertices to which $\mathrm{Z}$ gates are applied, while we connect two vertices by an edge where $\mathrm{CZ}$ gates are operated. Different graphs represent different graph states due to the commutative nature of the $\mathrm{Z}$ and the CZ gates. See Fig. 15(a2).

The set of all graph states is a subgroup of the REW states by the following reasoning. The number of the $\mathrm{Z}$ gates is $N$, while the number of the $\mathrm{CZ}$ gates is ${ }_{N} C_{2}$. Hence, we can express $2^{N+{ }_{N} C_{2}}$ graph states by using $\mathrm{Z}$ gates and $\mathrm{CZ}$ gates. On the other hand, the number of the REW states is $2^{2^{N}}$. Here, $2^{2^{N}}>2^{N+{ }_{N} C_{2}}$ for $N \geqslant 3$.

In order to represent a complete set of the REW states, it is necessary to introduce the notion of hypergraph [55,62], which is a generalization of graph. In a hypergraph, we have a hyperedge connecting more than three vertices. For example, a CCZ gate is represented by a hyperedge with order 3, which connects three vertices, as in Fig. 15(b2). In a similar way, a $\mathrm{C}^{p-1} \mathrm{Z}$ gate is represented by a hyperedge with order $p$, which connects $p$ vertices. The number of the $\mathrm{C}^{p-1} \mathrm{Z}$ gates is given

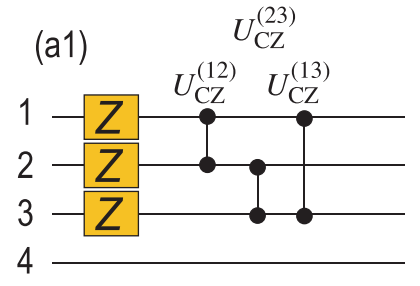

(a2)
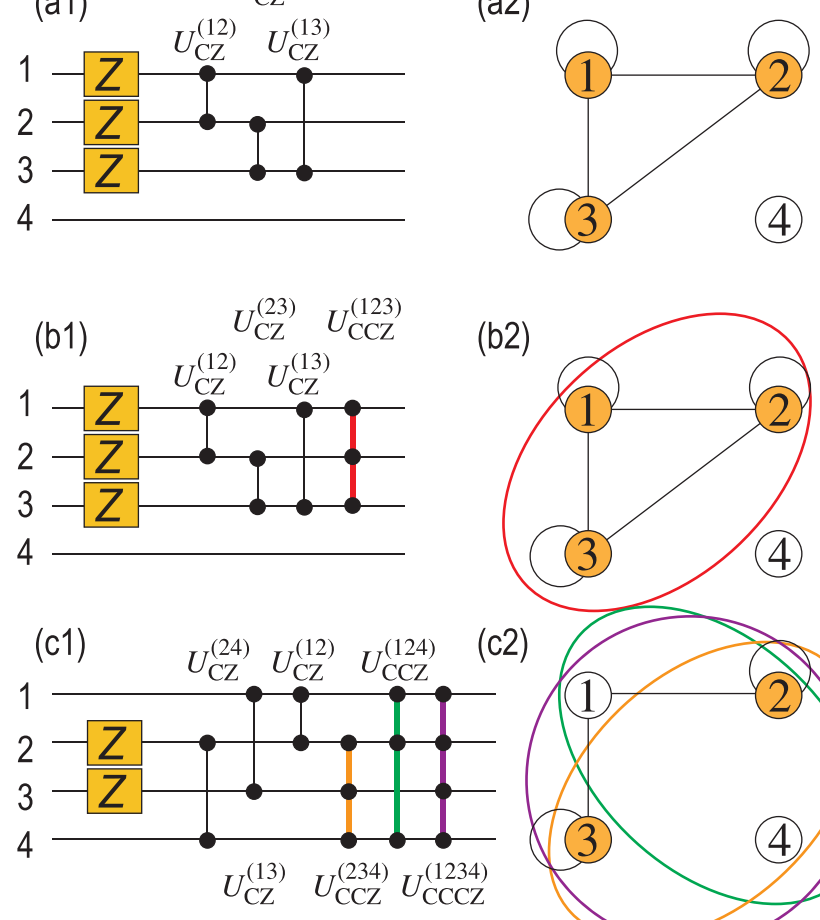

(c2)

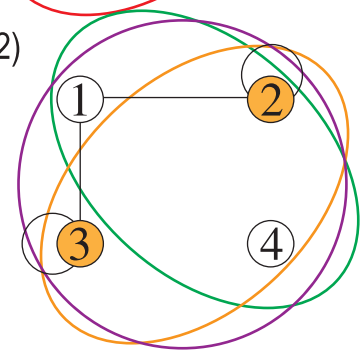

FIG. 15. (1) Quantum-circuit representation and (2) graph and hypergraph representation. $\mathrm{Z}$ gates correspond to self-loops, which are marked in orange disks in a graph or a hypergraph. $\mathrm{CZ}$ gates correspond to edges, which are marked in black lines in a hypergraph. $\mathrm{C}^{p-1} \mathrm{Z}$ gates correspond to hyperedges, which are marked in colored ovals in a hypergraph. (a) graph state, (b) for $\psi_{x}$, and (c) for $\psi_{w}$.

by ${ }_{N} C_{p}$. Then, the total number of gates is $2^{\sum_{p=1}^{N}\left({ }_{N} C_{p}\right)}=2^{2^{N}-1}$. The overall phase is irrelevant and thus it is a complete representation. We show a hypergraph representation of $\left|\psi_{x}\right\rangle$ and $\left|\psi_{w}\right\rangle$ in Fig. 15. In Fig. 15(b), a hyperedge is represented by an oval containing three vertices connected by a hyperedge. In Fig. 15(c), three hyperedges are represented by three ovals containing vertices connected by three hyperedge.

Weighted graph states and hypergraph states. As a generalization of graph states and hypergraph states, we may introduce the concepts of weighted graph states and weighted hypergraph states in the context of CEW states. A weighted graph state is defined by a sequential application of $\mathrm{Z}_{\phi}$ gates and $\mathrm{CZ}_{\phi}$ gates to the equal-coefficient state (69), where $e^{i \phi}$ is a weight. Next, a weighted hypergraph state is generated by a sequential application of $\mathrm{C}^{p-1} \mathrm{Z}_{\phi}$ gates to the equal-coefficient state (69). Here, we assign a $p$-hyperedge to a $C^{p-1} Z_{\phi}$ gate as in the case of $\mathrm{C}^{p-1} \mathrm{Z}$ gates, and then we assign a weight $e^{i \phi}$ to each hyperedge.

\section{DISCUSSION}

We have proposed an electric-circuit simulation of universal quantum gates on the basis of $L C$ resonators bridged by inductors. Here, capacitance and inductance are controllable by using a variable capacitance diode and an active inductor, respectively. The merit of the present scheme is that the system works at room temperature. It is highly contrasted with 
other methods including superconducting qubits and ion traps. In addition, it is compatible with a classical computer.

An artificial neuron requires many $\mathrm{C}^{p-1} \mathrm{Z}$ gates for various p. It is actually a hard task to realize $\mathrm{C}^{p-1} \mathrm{Z}$ gates in the standard quantum-circuit implementation even by employing modern technology such as superconductor, ion-trap or photonic systems for $p \geqslant 3$. This difficulty originates in the fact that a $\mathrm{C}^{p-1} \mathrm{Z}$ gate implies a $p$-body interaction. Although it is possible to decompose a $\mathrm{C}^{p-1} \mathrm{Z}$ gate into simpler gates, there emerge too many gates [3]. The problem becomes worse for a complex-artificial neuron, where we use $\mathrm{C}^{p-1} \mathrm{Z}_{\phi}$ gates instead of $\mathrm{C}^{p-1} \mathrm{Z}$ gates. The $\mathrm{C}^{p-1} \mathrm{Z}_{\phi}$ gate contains the phase-shift gate with angle $\phi$. It is possible but quite tedious to construct a $\mathrm{C}^{p-1} \mathrm{Z}_{\phi}$ gate with the use of a set of universal quantum gates. On the contrary, it is simple to construct a $\mathrm{C}^{p-1} \mathrm{Z}_{\phi}$ gate by inserting one $\phi$ phase-shift gate in the electric-circuit implementation.

Furthermore, it is a nontrivial problem to construct a superposition state such as REW or CEW states. It is necessary to design several quantum gates in order to make such a state, for which we need to use a classical computer in general. See a typical example in Fig. 13(a) and Appendix A. On the other hand, it is sufficient to insert simply some $\phi$ phase-shift gates in the electric-circuit implementation. Although the implementation of the Hadamard gates is harder, the assignment is systematic and trivial. See the corresponding example in Fig. 13(b).

We have previously proposed another kind of electriccircuit simulation of universal quantum gates, where quantum gates are constructed by bridging telegrapher wires $[13,14]$. The number of elements of electric circuits increases as the increase of the number of quantum gates. On the other hand, the number of the elements is fixed in the present scheme irrespective to the number of quantum gates because the operation is performed in time evolution. Another merit comparing to the wire construction $[13,14]$ is that the present scheme is programmable because the gate is applied temporally. It is contrasted to the wire construction, where the gates are constructed by setting wires.
The present scheme is not scalable, in which we need electric circuit elements in the order of $2^{N}$. In this sense, it is merely a simulator of a quantum computer. Needless to say, the matrix operation can be done by a classical computer. However, in a classical computer, a complex number is represented by a set of digital bits, which requires many bits for its accurate representation. On the contrary, the present system has the same mechanism as a quantum computer by simulating each quantum gate, where the complex number is directly represented by the amplitude and the phase of the voltage. Namely, it can simulate the direct evolution of complex numbers by unitary transformations. It will be useful for designing a future quantum computer.

We should also mention quantum-classical hybrid algorithms, to which much attention is recently paid. They work in a noisy-intermediate scale quantum computer (NISQ), which is a current quantum computer made of a small number of qubits without error correction. In such a hybrid system, however, a transformation of information is hard between classical and quantum computers. On the contrary, the present $L C$ resonator systems are compatible with a classical computer since both of them are made of electric circuits.

We have calculated fidelity due to randomness in electric circuit elements and the inaccuracy of time duration controlling the inductance. We find that the effect due the time inaccuracy is more serious than randomness in elements. It is because the voltage dynamics is sensitive to an inaccuracy of the time duration for controlling inductance. On the other hand, the fidelity is rather robust against randomness of elements in the order of $10 \%$, which are easily obtainable in markets.

\section{ACKNOWLEDGMENTS}

The author is very much grateful to E. Saito and N. Nagaosa for helpful discussions on the subject. This work is supported by the Grants-in-Aid for Scientific Research from MEXT KAKENHI (Grants No. JP17K05490 and No. JP18H03676). This work is also supported by CREST, JST (JPMJCR16F1 and JPMJCR20T2).

\section{APPENDIX A: EXAMPLE OF HYPERGRAPH GENERATION PROCESS}

A REW state $\left|\psi_{x}\right\rangle$ is given by Eq. (64) with $x_{j}= \pm 1$. We explain how to create this state from the equal-coefficient state (69). Alternatively, we explain how to transform this state to the equal-coefficient state.

Let us explicitly study an example given by

$$
\begin{aligned}
4\left|\psi_{x}\right\rangle= & -|0000\rangle-|0001\rangle+|0010\rangle+|0011\rangle+|0100\rangle+|0101\rangle+|0110\rangle+|0111\rangle \\
& +|1000\rangle+|1001\rangle+|1010\rangle+|1011\rangle+|1100\rangle+|1101\rangle+|1110\rangle+|1111\rangle .
\end{aligned}
$$

First, we rewrite it so that the coefficient of $|0000\rangle$ is 1 ,

$$
\begin{aligned}
4\left|\psi_{x}\right\rangle= & -(|0000\rangle+|0001\rangle-|0010\rangle-|0011\rangle-|0100\rangle-|0101\rangle-|0110\rangle-|0111\rangle \\
& -|1000\rangle-|1001\rangle-|1010\rangle-|1011\rangle-|1100\rangle-|1101\rangle-|1110\rangle-|1111\rangle) .
\end{aligned}
$$

We note that overall phase - is irrelevant in quantum computation. 
(i) We focus on the states $\left|n_{1} n_{2} n_{3} n_{4}\right\rangle$ such that $\sum_{i} n_{i}=1$, among which the coefficients of $|1000\rangle,|0100\rangle$ and $|0010\rangle$ are -1 , while the coefficient of $|0001\rangle$ is 1 . Then, we apply three $Z$ gates to the first, second and third qubits, and obtain

$$
\begin{aligned}
4 U_{\mathrm{Z}}^{(1)} U_{\mathrm{Z}}^{(2)} U_{\mathrm{Z}}^{(3)} \psi_{x}= & -(|0000\rangle+|0001\rangle+|0010\rangle+|0011\rangle+|0100\rangle+|0101\rangle-|0110\rangle-|0111\rangle \\
& +|1000\rangle+|1001\rangle-|1010\rangle-|1011\rangle-|1100\rangle-|1101\rangle+|1110\rangle+|1111\rangle)
\end{aligned}
$$

(ii) We focus on the states $\left|n_{1} n_{2} n_{3} n_{4}\right\rangle$ such that $\sum_{i} n_{i}=2$, among which the coefficients of $|0110\rangle,|1010\rangle$, and $|1100\rangle$ are -1 , while the coefficient of $|0011\rangle,|0101\rangle$, and $|1001\rangle$ is 1 . Then, we apply three $C Z$ gates, and obtain

$$
\begin{aligned}
4 U_{\mathrm{CZ}}^{(13)} U_{\mathrm{CZ}}^{(23)} U_{\mathrm{CZ}}^{(12)} U_{\mathrm{Z}}^{(1)} U_{\mathrm{Z}}^{(2)} U_{\mathrm{Z}}^{(3)} \psi_{x}= & -(|0000\rangle+|0001\rangle+|0010\rangle+|0011\rangle+|0100\rangle+|0101\rangle+|0110\rangle+|0111\rangle \\
& +|1000\rangle+|1001\rangle+|1010\rangle+|1011\rangle+|1100\rangle+|1101\rangle-|1110\rangle-|1111\rangle)
\end{aligned}
$$

(iii) We focus on the states $\left|n_{1} n_{2} n_{3} n_{4}\right\rangle$ such that $\sum_{i} n_{i}=3$, among which the coefficients of $|1110\rangle$ is -1 , while the coefficient of $|0111\rangle,|1011\rangle$, and $|1101\rangle$ is 1 . Then, we apply one $C C Z$ gate, and obtain the equal-coefficient state,

$$
\begin{aligned}
4 U_{\mathrm{CCZ}}^{(123)} U_{\mathrm{CZ}}^{(13)} U_{\mathrm{CZ}}^{(23)} U_{\mathrm{CZ}}^{(12)} U_{\mathrm{Z}}^{(1)} U_{\mathrm{Z}}^{(2)} U_{\mathrm{Z}}^{(3)} \psi_{x}= & -(|0000\rangle+|0001\rangle+|0010\rangle+|0011\rangle+|0100\rangle+|0101\rangle+|0110\rangle+|0111\rangle \\
& +|1000\rangle+|1001\rangle+|1010\rangle+|1011\rangle+|1100\rangle+|1101\rangle+|1110\rangle+|1111\rangle) .
\end{aligned}
$$

Hence, it follows from Eq. (71) that

$$
V_{x}^{-1}=-U_{\mathrm{CCZ}}^{(123)} U_{\mathrm{CZ}}^{(13)} U_{\mathrm{CZ}}^{(23)} U_{\mathrm{CZ}}^{(12)} U_{\mathrm{Z}}^{(1)} U_{\mathrm{Z}}^{(2)} U_{\mathrm{Z}}^{(3)} .
$$

Consequently, $\left|\psi_{x}\right\rangle$ is obtained by the inverse process as

$$
\psi_{x}=-U_{\mathrm{CCZ}}^{(123)} U_{\mathrm{CZ}}^{(13)} U_{\mathrm{CZ}}^{(23)} U_{\mathrm{CZ}}^{(12)} U_{\mathrm{Z}}^{(1)} U_{\mathrm{Z}}^{(2)} U_{\mathrm{Z}}^{(3)} \bigotimes_{s=1}^{4} U_{\mathrm{H}}^{(s)}|0000\rangle,
$$

because all $\mathrm{C}^{p-1} \mathrm{Z}$ gates are commutative and one $\mathrm{C}^{p-1} \mathrm{Z}$ gate $U$ satisfies $U^{2}=1$.

In a similar way,

$$
\begin{aligned}
\psi_{w}= & \frac{1}{4}(|0000\rangle+|0001\rangle-|0010\rangle-|0011\rangle-|0100\rangle+|0101\rangle+|0110\rangle+|0111\rangle \\
& +|1000\rangle+|1001\rangle+|1010\rangle+|1011\rangle+|1100\rangle+|1101\rangle+|1110\rangle+|1111\rangle)
\end{aligned}
$$

is generated as

$$
\psi_{w}=U_{\mathrm{CCCZ}}^{(1234)} U_{\mathrm{CCZ}}^{(124)} U_{\mathrm{CCZ}}^{(234)} U_{\mathrm{CZ}}^{(12)} U_{\mathrm{CZ}}^{(13)} U_{\mathrm{CZ}}^{(24)} U_{\mathrm{Z}}^{(2)} U_{\mathrm{Z}}^{(3)} \bigotimes_{s=1}^{4} U_{\mathrm{H}}^{(s)}|0000\rangle .
$$

Thus, it is a nontrivial problem to construct hypergraph generation circuits in the standard quantum-circuit implementation.

\section{APPENDIX B: NUMBER RECOGNITION}

We show binary representations of the pattern of each number for the reference and the input data in Fig. 9, where the left-hand side stands for the reference and the right one for the input data,

$$
\begin{array}{lll}
0:(11111001100110011111), & (01101001100110010110), \\
1:(01101010001000101111), & (01100010001000100010), \\
2:(11111001001001001111), & (01110001001001000111), \\
3:(01101001001010010110), & (11111001001010011111), \\
4:(10101010111100100010), & (00101010111000100010), \\
5:(11111000111100011111), & (01101000111000011110), \\
6:(11111000111110011111), & (01101000111010011110), \\
7:(11110001001000100100), & (01100001001000100010), \\
8:(11111001111110011111), & (01101001011010010110), \\
9:(11111001111100011111), & (01101001011100010110),
\end{array}
$$

where 0 indicates a white pixel and 1 indicates a black pixel. 
[1] R. Feynman, Simulating physics with computers, Int. J. Theor. Phys. 21, 467 (1982).

[2] D. P. DiVincenzo, Quantum computation, Science 270, 255 (1995).

[3] M. Nielsen and I. Chuang, Quantum Computation and Quantum Information (Cambridge University Press, 2016).

[4] Y. Nakamura, Yu. A. Pashkin, and J. S. Tsai, Coherent control of macroscopic quantum states in a single-Cooper-pair box, Nature (London) 398, 786 (1999).

[5] E. Knill, R. Laflamme, and G. J. Milburn, A scheme for efficient quantum computation with linear optics, Nature (London) 409, 46 (2001).

[6] D. Loss and D. P. DiVincenzo, Quantum computation with quantum dots, Phys. Rev. A 57, 120 (1998).

[7] J. I. Cirac and P. Zoller, Quantum Computations with Cold Trapped Ions, Phys. Rev. Lett. 74, 4091 (1995).

[8] L. M. K. Vandersypen, M. Steffen, G. Breyta, C. S. Yannoni, M. H. Sherwood, and I. L. Chuang, Experimental realization of Shor's quantum factoring algorithm using nuclear magnetic resonance, Nature (London) 414, 883 (2001).

[9] B. E. Kane, A silicon-based nuclear spin quantum computer, Nature (London) 393, 133 (1998).

[10] D. Deutsch, Quantum theory, the Church-Turing principle and the universal quantum computer, Proc. R. Soc. A 400, 97 (1985).

[11] C. M. Dawson and M. A. Nielsen, The Solovay-Kitaev algorithm, arXiv:quant-ph/0505030.

[12] M. Nielsen and I. Chuang, Quantum Computation and Quantum Information (Cambridge University Press, Cambridge, UK, 2010).

[13] M. Ezawa, Electric circuits for universal quantum gates and quantum Fourier transformation, Phys. Rev. Research 2, 023278 (2020).

[14] M. Ezawa, Dirac formulation for universal quantum gates and shor's integer factorization in high-frequency electric circuits, J. Phys. Soc. Jpn. 89, 124712 (2020).

[15] M. Ezawa, Non-Abelian braiding of Majorana-like edge states and topological quantum computations in electric circuits, Phys. Rev. B 102, 075424 (2020).

[16] C. H. Lee, S. Imhof, C. Berger, F. Bayer, J. Brehm, L. W. Molenkamp, T. Kiessling, and R. Thomale, Topolectrical circuits, Commun. Phys. 1, 39 (2018).

[17] S. Imhof, C. Berger, F. Bayer, J. Brehm, L. Molenkamp, T. Kiessling, F. Schindler, C. H. Lee, M. Greiter, T. Neupert, and R. Thomale, Topolectrical-circuit realization of topological corner modes, Nat. Phys. 14, 925 (2018).

[18] M. Serra-Garcia, R. Susstrunk, and S. D. Huber, Observation of quadrupole transitions and edge mode topology in an LC circuit network, Phys. Rev. B 99, 020304(R) (2019).

[19] T. Helbig, T. Hofmann, C. H. Lee, R. Thomale, S. Imhof, L. W. Molenkamp, and T. Kiessling, Band structure engineering and reconstruction in electric circuit networks, Phys. Rev. B 99, 161114(R) (2019).

[20] E. I. Rosenthal, N. K. Ehrlich, M. S. Rudner, A. P. Higginbotham, and K. W. Lehnert, Topological phase transition measured in a dissipative metamaterial, Phys. Rev. B 97, 220301(R) (2018).

[21] Y. Lu, N. Jia, L. Su, C. Owens, G. Juzeliunas, D. I. Schuster, and J. Simon, Probing the Berry curvature and Fermi arcs of a Weyl circuit, Phys. Rev. B 99, 020302(R) (2019).
[22] M. Ezawa, Higher-order topological electric circuits and topological corner resonance on the breathing kagome and pyrochlore lattices, Phys. Rev. B 98, 201402(R) (2018).

[23] Y. Li, Y. Sun, W. Zhu, Z. Guo, J. Jiang, T. Kariyado, H. Chen, and $\mathrm{X}$. Hu, Topological LC-circuits based on microstrips and observation of electromagnetic modes with orbital angular momentum, Nat. Commun. 9, 4598 (2018).

[24] T. Hofmann, T. Helbig, C. H. Lee, M. Greiter, and R. Thomale, Chiral Voltage Propagation and calibration in a topolectrical chern circuit, Phys. Rev. Lett. 122, 247702 (2019).

[25] K. Luo, R. Yu, and H. Weng, Topological Nodal States in Circuit Lattice, Research 2018, 6793752 (2018).

[26] M. Ezawa, Non-Hermitian higher-order topological states in nonreciprocal and reciprocal systems with their electric-circuit realization, Phys. Rev. B 99, 201411(R) (2019).

[27] M. Ezawa, Non-Hermitian higher-order topological states in nonreciprocal and reciprocal systems with their electric-circuit realization, Phys. Rev. B 99, 121411(R) (2019).

[28] M. Ezawa, Braiding of Majorana-like corner states in electric circuits and its non-Hermitian generalization, Phys. Rev. B 100, 045407 (2019).

[29] T. Helbig, T. Hofmann, S. Imhof, M. Abdelghany, T. Kiessling, L. W. Molenkamp, C. H. Lee, A. Szameit, M. Greiter, and R. Thomale, Generalized bulk-boundary correspondence in nonHermitian topolectrical circuits, Nat. Phys. 16, 747 (2020).

[30] M. Ezawa, Electric-circuit simulation of the Schrodinger equation and non-Hermitian quantum walks, Phys. Rev. B 100, 165419 (2019).

[31] S. Lloyd, M. Mohseni, and P. Rebentrost, Quantum algorithms for supervised and unsupervised machine learning, arXiv:1307.0411.

[32] M. Schuld, I. Sinayskiy, and F. Petruccione, An introduction to quantum machine learning, Contemp. Phys. 56, 172 (2014).

[33] J. Biamonte, P. Wittek, N. Pancotti, P. Rebentrost, N. Wiebe, and S. Lloyd, Quantum machine learning, Nature (London) 549, 195 (2017).

[34] P. Wittek, Quantum Machine Learning: What Quantum Computing Means to Data Mining (Academic Press, 2014).

[35] A. W. Harrow, A. Hassidim, and S. Lloyd, Quantum Algorithm for Linear Systems of Equations, Phys. Rev. Lett. 103, 150502 (2009).

[36] N. Wiebe, D. Braun, and S. Lloyd, Quantum Algorithm for Data Fitting, Phys. Rev. Lett. 109, 050505 (2012).

[37] P. Rebentrost, M. Mohseni, and S. Lloyd, Quantum Support Vector Machine for Big Data Classification, Phys. Rev. Lett. 113, 130503 (2014).

[38] Z. Li, X. Liu, N. Xu, and J. Du, Experimental Realization of a Quantum Support Vector Machine, Phys. Rev. Lett. 114, 140504 (2015).

[39] M. Schuld and N. Killoran, Quantum Machine Learning in Feature Hilbert Spaces, Phys. Rev. Lett. 122, 040504 (2019).

[40] V. Havlicek, A. D. Corcoles, K. Temme, A. W. Harrow, A. Kandala, J. M. Chow, and J. M. Gambetta, Supervised learning with quantum-enhanced feature spaces, Nature (London) 567, 209 (2019)

[41] L. Lamata, Mach. Learn.: Sci. Technol. 1, 033002 (2020).

[42] I. Cong, S. Choi, and M. D. Lukin, Quantum convolutional neural networks, Nat. Phys. 15, 1273 (2019).

[43] J. Schmidhuber, Deep learning in neural networks: An overview, Neural Networks 61, 85 (2015). 
[44] J. M. Zurada, Introduction to Artificial Neural Systems (West Group, St. Paul, MN, USA, 1992).

[45] M. Schuld, I. Sinayskiy, and F. Petruccione, Simulating a perceptron on a quantum computer, Phys. Lett. A 379, 660 (2015).

[46] N. Wiebe, A. Kapoor, and K. N. Svore, Quantum Perceptron Models, arXiv:1602.04799.

[47] Y. Cao, G. G. Guerreschi, and A. Aspuru-Guzik, Quantum Neuron: an elementary building block for machine learning on quantum computers, arXiv:1711.11240.

[48] F. Tacchino, C. Macchiavello, D. Gerace, and D. Bajoni, An artificial neuron implemented on an actual quantum processor, npj Quantum Inf. 5, 26 (2019).

[49] E. Torrontegui and J. J. Garcia-Ripoll, Unitary quantum perceptron as efficient universal approximator, Europhys. Lett. 125, 30004 (2019).

[50] L. B. Kristensen, M. Degroote, P. Wittek, A. Aspuru-Guzik, and N. T. Zinner, An Artificial Spiking Quantum Neuron, arXiv:1907.06269.

[51] N. Killoran, T. R. Bromley, J. M. Arrazola, M. Schuld, N. Quesada, and S. Lloyd, Continuous-variable quantum neural networks, Phys. Rev. Research 1, 033063 (2019).

[52] S. Mangini, F. Tacchino, D. Gerace, C. Macchiavello, and D. Bajoni, Quantum computing model of an artificial neuron with continuously valued input data, Mach. Learn.: Sci. Technol. 1, 045008 (2020).

[53] D. Deutsch and R. Jozsa, Rapid solution of problems by quantum computation, Proc. R. Soc. London A 439, 553 (1992).
[54] L. Grover, A fast quantum mechanical algorithm for database search, Proceedings of the Twenty-Eighth Annual ACM Symposium on Theory of Computing (ACM, 1996), p. 212.

[55] M. Rossi, M. Huber, D. Bruss, and C. Macchiavello, Quantum hypergraph states, New J. Phys. 15, 113022 (2013).

[56] F. Rosenblatt, The Perceptron: A Perceiving and Recognizing Automaton, Tech. Rep. Inc. Report No. 85-460-1, Cornell Aeronautical Laboratory (1957).

[57] W. S. McCulloch and W. Pitts, A logical calculus of the ideas immanent in nervous activity, Bull. Math. Biophys. 5, 115 (1943).

[58] X. Glorot, A. Bordes, and Y. Bengio, Deep Sparse Rectifier Neural Networks, Proceedings of the Fourteenth International Conference on Artificial Intelligence and Statistics (AISTATS11) $15: 315$ (2011).

[59] M. Hein, J. Eisert, and H. J. Briegel, Multiparty entanglement in graph states, Phys. Rev. A 69, 062311 (2004).

[60] M. Hein, W. Dur, J. Eisert, R. Raussendorf, M. Van den Nest, and H.-J. Briegel, Entanglement in graph states and its applications, Proceedings of the International School of Physics "Enrico Fermi" on "Quantum Computers, Algorithms and Chaos” (IOS Press, 2005).

[61] S. Anders and H. J. Briegel, Fast simulation of stabilizer circuits using a graph-state representation, Phys. Rev. A 73, 022334 (2006).

[62] R. Qu, J. Wang, Z.-S. Li, and Y.-R. Bao, Encoding hypergraphs into quantum states, Phys. Rev. A 87, 022311 (2013). 